OPEN ACCESS

Edited by:

Kongkai Zhu,

University of Jinan, China

Reviewed by:

Hiromi Sakai,

Nara Medical University, Japan

Roland Pittman,

Virginia Commonwealth University,

United States

Andrea Mozzarelli,

University of Parma, Italy

*Correspondence:

Xiaobo Huang

drhuangxb@163.com

Dingding Zhang

zhangdd25@126.com

Specialty section:

This article was submitted to

Experimental Pharmacology and Drug

Discovery,

a section of the journal

Frontiers in Pharmacology

Received: 17 August 2021 Accepted: 10 November 2021

Published: 30 November 2021

Citation:

Cao M, Wang G, He H, Yue R, Zhao Y, Pan L, Huang W, Guo Y, Yin T, Ma L,

Zhang $D$ and Huang $X$ (2021)

Hemoglobin-Based Oxygen Carriers:

Potential Applications in Solid

Organ Preservation.

Front. Pharmacol. 12:760215.

doi: 10.3389/fphar.2021.760215

\section{Hemoglobin-Based Oxygen Carriers: Potential Applications in Solid Organ Preservation}

\author{
Min $\mathrm{Cao}^{1}$, Guoqing Wang ${ }^{1}$, Hongli He ${ }^{1}$, Ruiming Yue ${ }^{1}$, Yong Zhao ${ }^{2}$, Lingai Pan ${ }^{1}$, \\ Weiwei Huang ${ }^{1}$, Yang Guo ${ }^{1}$, Tao Yin ${ }^{3}$, Lina Ma ${ }^{4}$, Dingding Zhang ${ }^{5 *}$ and Xiaobo Huang ${ }^{1 *}$ \\ ${ }^{1}$ Department of Critical Care Medicine, Sichuan Provincial People's Hospital, University of Electronic Science and Technology of \\ China, Chengdu, China, ${ }^{2}$ Anesthesiology, Southwest Medicine University, Luzhou, China, ${ }^{3}$ Surgical Department, Chengdu \\ Second People's Hospital, Chengdu, China, ${ }^{4}$ Health Inspection and Quarantine, Chengdu Medical College, Chengdu, China, \\ ${ }^{5}$ Sichuan Provincial Key Laboratory for Disease Gene Study, Sichuan Provincial People's Hospital, University of Electronic \\ Science and Technology of China, Chengdu, China
}

Ameliorating graft injury induced by ischemia and hypoxia, expanding the donor pool, and improving graft quality and recipient prognosis are still goals pursued by the transplant community. The preservation of organs during this process from donor to recipient is critical to the prognosis of both the graft and the recipient. At present, static cold storage, which is most widely used in clinical practice, not only reduces cell metabolism and oxygen demand through low temperature but also prevents cell edema and resists apoptosis through the application of traditional preservation solutions, but these do not improve hypoxia and increase oxygenation of the donor organ. In recent years, improving the ischemia and hypoxia of grafts during preservation and repairing the quality of marginal donor organs have been of great concern. Hemoglobin-based oxygen carriers (HBOCs) are "made of" natural hemoglobins that were originally developed as blood substitutes but have been extended to a variety of hypoxic clinical situations due to their ability to release oxygen. Compared with traditional preservation protocols, the addition of HBOCs to traditional preservation protocols provides more oxygen to organs to meet their energy metabolic needs, prolong preservation time, reduce ischemia-reperfusion injury to grafts, improve graft quality, and even increase the number of transplantable donors. The focus of the present study was to review the potential applications of HBOCs in solid organ preservation and provide new approaches to understanding the mechanism of the promising strategies for organ preservation.

Keywords: hemoglobin-based oxygen carriers, solid organ preservation, machine perfusion, organ oxygenation, ischemia-reperfusion injury

\section{INTRODUCTION}

Solid organ transplantation (SOT) is an optimal, lifesaving treatment choice for patients with endstage organ failure. The number of transplants has increased dramatically in the last decade, but there remains a considerable imbalance between the demand and supply of donor organs worldwide (OPTN/SRTR 2019 Annual Data Report, 2021a, 2021b). According to the data from the OPTN/ SRTR 2019 Annual Data Report about Deceased Organ Donation, donations after circulatory death (DCD) increased to 32,313 in 2019 from 29,675 in 2018 (Israni et al., 2021). Ischemia-reperfusion 
injury (IRI) unavoidably occurs during organ resection and transplantation, may compromise the short-term and longterm after transplantation, and remains a critical organ transplantation challenge. With the increase in expanded criteria donor (ECD), the selection of better preservation methods to improve tissue oxygenation during isolated organ preservation to further reduce IRI of organs remains a pressing issue.

Ischemia and hypoxia are the leading causes of graft damage during the storage of isolated organs and may lead to a variety of destructive effects, such as ion imbalance, uncoupling of mitochondria, activation of endothelial cells, activation of various cell death programs, and proinflammatory immune responses (Kalisvaart et al., 2018; Lavallard et al., 2020). The current static cold storage (SCS) of grafts reduces enzyme activity and oxygen consumption, but the demand for energy metabolism and oxygen continues while cells maintain ion homeostasis and membrane potential (Kasil et al., 2019; Ali et al., 2020). Several methods for supplementing oxygen in organ preservation solutions have been reported. One method is the direct infusion of gaseous oxygen through mechanical perfusion platforms using various techniques, such as retrograde perfusion and supplemental oxygen under high pressure conditions (Higuchi et al., 2006; Minor et al., 2011; Tran et al., 2012). Supplemental oxygen during organ preservation promotes mitochondrial adenosine triphosphate (ATP) synthesis, prevents anaerobic metabolism, and reduces reperfusion injury (Minor et al., 2011). However, if the oxidative metabolism of the graft is severely impaired, direct oxygenation may exacerbate the oxidative stress damage to the organ. Another approach is to use oxygen carriers such as red blood cells (RBCs), humans, animals, recombinant sources, and perfluorochemicals (PFCs) (Tsujimura et al., 2002; Sakai et al., 2011; Teh et al., 2017; Liu et al., 2021). Perfusion of organs with blood as an oxygen carrier has been shown to reduce IRI to organs and improve grafts' quality and short-term prognosis. However, blood has some potential disadvantages, including immune phenomena, blood infection, cross-matching and scarcity, and it can only be used for subnormothermic machine perfusion (SNMP) and normothermic machine perfusion (NMP) because RBCs can undergo hemolysis in a low-temperature environment (Cai et al., 2020; Lv et al., 2021). PFCs are hydrophobic, lipophilic, and difficult to disinfect, making it difficult to promote their use in clinical applications (Partlow et al., 2008). Therefore, researchers have been trying to find solutions that can provide oxygen close to physiological conditions during the preservation of isolated organs. Hemoglobin-based oxygen carriers (HBOCs) are a class of oxygen carriers that bind and release oxygen closer to physiological conditions than that of perfluorocarbons.

HBOCs are cell-free carriers obtained by processing free hemoglobin via chemical modification, genetic modification, or embedding (Standl et al., 2003). The oxygen affinity of some HBOCs is lower than that of human hemoglobin, thus facilitating oxygen release to tissues as the hemoglobin dissociation curve shifts to the right (Page et al., 1998). HBOCs were initially investigated as a blood substitute and have now been expanded for ischemic and hypoxic treatments. The application of HBOCs to the preservation of isolated organs may have several potential advantages: maintenance of cellular metabolic needs and mitigation of hypoxic damage; much lower diameter than that of RBCs for better oxygen delivery; no immune reactivity; the needs of the period of organ preservation; good stability for long storage time; wide range of temperature adaptation; and easy availability from a wide variety of sources. The effectiveness and safety of HBOCs in the preservation of grafts have been studied in numerous preclinical and clinical studies. This review summarizes the characteristics of HBOCs and presents recent advances in the preservation of solid organs in preclinical and clinical studies with the addition of HBOCs to the preservation solution.

\section{OVERVIEW OF HBOCS}

HBOCs are biological products obtained from purified hemoglobin $(\mathrm{Hb})$ to have the ability to bind and release oxygen. $\mathrm{Hb}$ is a tetrameric protein molecule of $\sim 64 \mathrm{kDa}$ consisting of two noncovalently bound $\alpha \beta$ dimers (Sahu et al., 2006). Free $\mathrm{Hb}$ released from aged or damaged RBCs dissociates in dimers that in plasma have a half-life only of a few hours (Bleeker et al., 1989). The released heme causes lipid peroxide- or $\mathrm{H}_{2} \mathrm{O}_{2}$-driven formation of ferryl myoglobin $(\mathrm{Mb})$, which generates radicals and contributes to renal dysfunction (Moore et al., 1998; Alayash et al., 2007). Moreover, dimers are ultrafiltered in the kidneys, causing nephrotoxicity (Simoni et al., 1997). Hb cross-linking, polymerization, and conjugation to polymers prolong intravascular half-life, stability, and safety (Adachi et al., 1987; Bobofchak et al., 2003; Gordon et al., 2005). HBOCs were initially developed as blood substitutes. Numerous preclinical and clinical studies initially explored the safety and efficacy of HBOCs. HBOC201 has been available in South Africa since 2001 and is approved for surgical patients with acute anemia (Mer et al., 2016). However, in 2008, Dr. Natanson and others included 13 randomized controlled trials in a meta-analysis of cell-free hemoglobin-based blood substitutes and concluded that the use of HBOCs was associated with a significantly increased risk of death and myocardial infarction (MI) based on an analysis of the available data (Natanson et al., 2008). Subsequently, the Food and Drug Administration (FDA) suspended all HBOC trials in the United States. The study of this meta-analysis generated widespread controversy. Related editorials suggested that if the imminent risk of death due to low $\mathrm{Hb}$ outweighed the risk of HBOCs, requiring a moratorium on all HBOC trials could be fatal for these patients (Levien et al., 2008; Sarani and Gracias, 2008; Sauaia et al., 2008). Since 2014, clinical trials for HBOCs are currently available only through expanded access (EA) to save patients' lives when other interventions are not available (Mackenzie et al., 2010). HBOCs are presently approved for veterinary use in the United States, Russia, and the European Union (Jahr et al., 2008). Owing to the ability to bind oxygen and release oxygen, HBOCs have been extended for applications such as protection of isolated organs (van Leeuwen et al., 2019), initiation of 
TABLE 1 | Overview of different oxygen carriers in solid organ preservation.

\begin{tabular}{|c|c|c|c|c|c|c|c|c|c|}
\hline Product & $\begin{array}{l}\text { Current } \\
\text { status }\end{array}$ & Company & $\begin{array}{l}\text { Source } \\
\text { of } \mathrm{Hb}\end{array}$ & Modification & Half-life & Size & $\begin{array}{l}\text { Average } \\
\text { particle } \\
\text { diameter }\end{array}$ & $\mathbf{P}_{50}$ & $\begin{array}{l}\text { Shelf } \\
\text { life }\end{array}$ \\
\hline M101 & Clinical & $\begin{array}{l}\text { Hemarina, Morlaix, } \\
\text { Brittany, France }\end{array}$ & $\begin{array}{l}\text { Marine } \\
\text { Isnvertebrate } \\
\text { (Arenicola marina) }\end{array}$ & $\begin{array}{l}\text { Hexagonal bilayer- } \\
\text { linked globin } \\
\text { molecules }\end{array}$ & 2.5 days & $3,600 \mathrm{kDa}$ & $25 \mathrm{~nm}$ & $7 \mathrm{mmHg}$ & $\mathrm{N}$ \\
\hline HBOC-201 & Clinical & $\begin{array}{l}\text { Acquired by Hemoglobin } \\
\text { Oxygen Therapeutics in } \\
\text { 2014, Souderton, PA }\end{array}$ & Bovine & $\begin{array}{l}\text { Glutaraldehyde } \\
\text { polymerization }\end{array}$ & $19-24 \mathrm{~h}$ & $250 \mathrm{kDa}$ & $8 \mathrm{~nm}$ & $40 \mathrm{mmHg}$ & 3 years \\
\hline $\mathrm{HbV}$ & Preclinical & $\begin{array}{l}\text { Waseda Univ. \& Keio Univ; } \\
\text { Nara Medical University } \\
\text { (2013) }\end{array}$ & Human & $\begin{array}{l}\text { Polyethylene glycol } \\
\text { (PEG) chains }\end{array}$ & $15-20$ & $\mathrm{~N}$ & $250 \mathrm{~nm}$ & $9 \mathrm{mmHg}$ & 2 years \\
\hline polypHb & Experimental & $\begin{array}{l}\text { Tianjin Union Stem Cell } \\
\text { Genetic Engineering Ltd., } \\
\text { Tianjin, China }\end{array}$ & Human & $\begin{array}{l}\text { Cross-linkage with } \\
\text { glutaraldehyde }\end{array}$ & $\mathrm{N}$ & $64-600 \mathrm{kDa}$ & $N$ & $5-9 \mathrm{mmHg}$ & $\mathrm{N}$ \\
\hline HbG-200 & Preclinical & $\begin{array}{l}\text { Oxyglobin }^{\circledR} \text {, Biopure, } \\
\text { Cambridge, MA }\end{array}$ & Bovine & $\begin{array}{l}\text { Glutaraldehyde- } \\
\text { polymerized }\end{array}$ & $18-43 h$ & $200 \mathrm{kDa}$ & $N$ & $34 \mathrm{mmHg}$ & $\mathrm{N}$ \\
\hline $\begin{array}{l}\text { Human red } \\
\text { blood cells }\end{array}$ & Clinical & $\mathrm{N}$ & Human & $\mathrm{N}$ & 120 days & $64 \mathrm{KDa}$ & $7000 \mathrm{~nm}$ & $27 \mathrm{mmHg}$ & 3 weeks \\
\hline
\end{tabular}

$N$, not reported; HbV, hemoglobin-vesicles; PolyPHb, polymerized human placenta hemoglobin; HbG, hemoglobin glutamer.

extracorporeal membrane oxygenation (ECMO) circuit as the pump-priming solution (York et al., 2002; Henderson et al., 2004), tumors (Belcher et al., 2020), and other ischemic and hypoxic diseases.

Based on the different sources of $\mathrm{Hb}$, the main HBOCs applied for organ preservation are marine lugworm Arenicola marina (M101) (Arenicola marina), HBOC-201 (bovine), hemoglobinvesicles (HbV) (human), polymerized human placenta hemoglobin (PolyPHb) (human), and hemoglobin glutamer (HbG) 200 (bovine). An overview of the different oxygen carriers used in solid organ preservation is provided to understand their properties better (Table 1). HBOCs of different origins have commonalities and dissimilarities. HBOCs have the advantages of no cell membrane, no blood type, no cross-matching requirements, no risk of viral infection, long-term storage, and timely availability, so they are excellent options for the preservation of isolated organs (Caswell et al., 2005). The molecular diameters of several HBOCs used for organ preservation $(8-250 \mathrm{~nm})$ are much smaller than those of RBCs $(7,000 \mathrm{~nm})$ and their viscosity is lower, thus making it easier for HBOCs not only to enter tissues through blood vessels and improve tissue oxygenation but also to promote oxygen diffusion in the microcirculation, resulting in a uniform distribution in the graft (Scatena and Giardina, 2001). Due to the different sources of $\mathrm{Hb}$, different HBOCs also have their unique characteristics. M101 is a natural extracellular biopolymer $\mathrm{Hb}$ obtained from a marine invertebrate, Arenicola marina. M101 has a $3,600-\mathrm{kDa}$ structure composed of 156 globins and 44 nonglobin linker chains that can carry up to $156 \mathrm{O}_{2}$ molecules when saturated (Toulmond et al., 1990; Zal et al., 1997; Rousselot et al., 2006). M101 possesses intrinsic $\mathrm{Cu} / \mathrm{Zn}$-superoxide dismutase antioxidant activity, protecting tissue from superoxide radicals to a certain extent (Rousselot, et al., 2006). M101 is formulated as a good manufacturing practice (GMP)-compliant commercial class III medical device that is used as an additive to existing organ preservation solutions.
HBOC-201 [hemoglobin glutamer-250 (bovine); Hemopure, $\mathrm{HbO}_{2}$ Therapeutics LLC, Souderton, PA 18964, United States] is derived from bovine $\mathrm{RBCs}$. Free $\mathrm{Hb}$ is then purified by chromatography and cross-linked with glutaraldehyde (GDA) to increase the stability and molecular size (Hughes et al., 1995; Callas et al., 1997). When fully saturated, HBOC-201 binds approximately $1.36 \mathrm{ml}$ oxygen per gram of $\mathrm{Hb}$. Since the $\mathrm{Hb}$ dissociation curve is shifted to the right, it releases oxygen more easily than human $\mathrm{Hb}$ (Callas et al., 1997; Dubé et al., 2008). PolyPHb is purified from fresh human placental blood, heattreated to inactivate it, and then intramolecularly and intermolecularly cross-linked with pyridoxal phosphate (PLP) and GDA. Then, ultrafiltration and molecular sieve chromatography are performed to obtain polyhemoglobin with a molecular weight of $64-600 \mathrm{kDa}$ (Li et al., 2009). The $\mathrm{HbV}$ is a cellular-type HBOC involving polyethylene glycol (PEG), in which phospholipid vesicles encapsulating nearly 35-40 g/dl human $\mathrm{Hb}$ are embedded; the $\mathrm{Hb}$ concentration of the $\mathrm{HbV}$ suspension is $10 \mathrm{~g} / \mathrm{dl}$. One $\mathrm{HbV}$ particle ( $250 \mathrm{~nm}$ diameter) contains approximately 30,000 $\mathrm{Hb}$ molecules (Sakai et al., 1996; Sakai et al., 2000; Sakai et al., 2001).

According to previous studies, direct infusion of HBOCs in the body can cause vasoconstriction, methemoglobin, and other side effects. The causes of vasoconstriction may include the following factors: scavenge nitric oxide (NO) (Standl, 2005), upregulated endothelin (ET) production (Taverne et al., 2017), the low viscosity and shear stress (Pearce and Gawryl, 2003), and vessel wall hyperoxygenation (Standl, 2005; Cabrales et al., 2009), of which the scavenging of NO is the leading cause of vasoconstriction. $\mathrm{Hb}$ in $\mathrm{RBCs}$ not only binds and transports oxygen in the circulation but also is an efficient scavenger of NO (Doyle and Hoekstra, 1981). However, the NO-scavenging rate of $\mathrm{Hb}$ in $\mathrm{RBCs}$ is reduced by a red cell-free zone along the endothelium in the blood, extracellular diffusion of NO to RBCs, and reduced NO diffusion over RBC membrane. All three of these mechanisms are eliminated by direct intravascular infusion of 
HBOCs, and the high affinity and low shear stress of HBOCs for NO also lead to a reduction in circulating NO (Jeffers et al., 2006). Thus, in the presence of free $\mathrm{Hb}$, bioavailable NO is reduced and leads to vasoconstriction and hypertension, increased proinflammatory mediators and vasoconstrictive factors, and platelet inactivation (Loscalzo, 1997; Rother et al., 2005).

Although there are differences in these vascular parameters, there are few reports on vasoconstriction damage in the preservation of isolated organs. The following reasons may underlie the relative lack of reports: carbon monoxide (CO) production is increased during the degradation of heme (Alayash, 2017; Zhu et al., 2019); HBOCs provide a sufficient oxygen supply to cells, which may balance the effects of vascular parameters (Gould et al., 1998); the concentration of HBOCs during the preservation of isolated organs is much lower (de Vries et al., 2019); compared to protecting isolated organs from ischemia-reperfusion injury, the advantages of HBOCs outweigh the disadvantages (Topp et al., 2008); the lavage fluid neutralizes the HBOCs before they reach the recipient; and the concentration of HBOCs that eventually reaches the circulation is negligible (Mahboub et al., 2020). Unlike RBCs, HBOCs do not contain NADH-dependent methemoglobin reductase, which is responsible for converting methemoglobin back to $\mathrm{Hb}$. The reversible oxygen binding of $\mathrm{Hb}$ to oxygen depends on the reduced state of iron atoms in the structure of $\mathrm{Hb}$. However, one or more iron ions in methemoglobin are in the oxidized state and are unable to bind oxygen while increasing the affinity of $\mathrm{Hb}$ for oxygen and shifting the oxygen dissociation curve to the left, resulting in decreasing its ability to release and deliver oxygen (Dixit et al., 2021). However, elevated methemoglobin in the recipient has rarely been reported when HBOCs are applied to preserve grafts. This is possible because methemoglobin is washed out of the perfusion fluid before transplantation, making the amount of methemoglobin that eventually reaches the recipient's circulation negligible. Moreover, supplementation with additional HBOC-201, glutathione, vitamin $\mathrm{C}$, and methylene blue also corrected or slowed the increased percentage of methemoglobin caused by HBOCs (de Vries et al., 2019; Mackenzie et al., 2010; Unnikrishnan et al., 2019). Therefore, applying conventional preservation solutions with added HBOCs to the preservation of isolated organs can adequately improve the oxygenation of tissues with fewer side effects. More importantly, more randomized clinical trials with large samples are needed to validate the safety and efficacy of HBOCs in SOT.

\section{HBOCS IN SOLID ORGAN TRANSPLANTATION}

The preservation of isolated organs is necessary before transplantation into a recipient's body. Thus, the choice of preservation solution is crucial for the protection of isolated organs. Countless traditional preservation solutions were developed with the increase in SOT in the 1970s, such as Euro-Collins (EC) (Ingemansson et al., 1995), Celsior, University of Wisconsin (UW), and histidine-tryptophan-

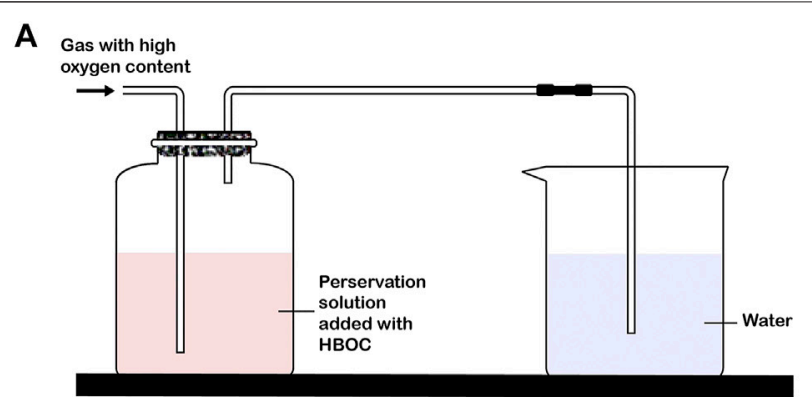

B

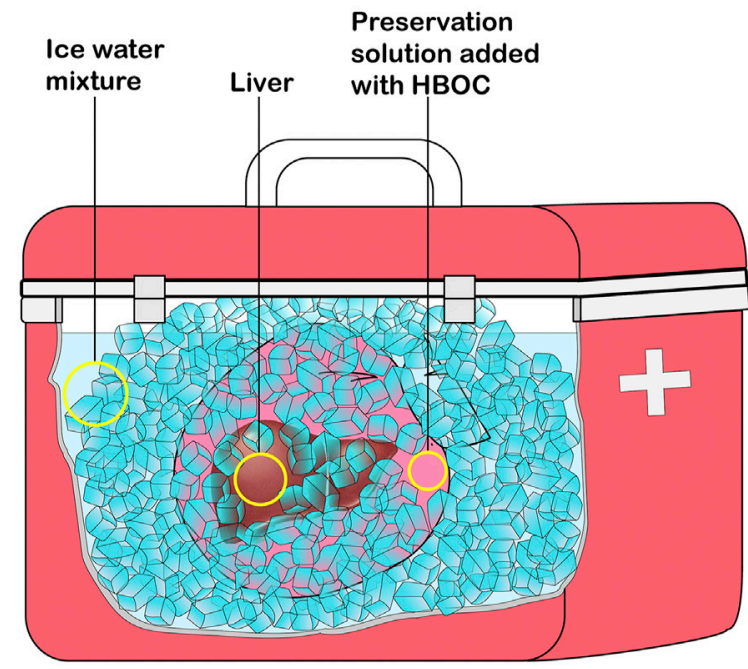

C

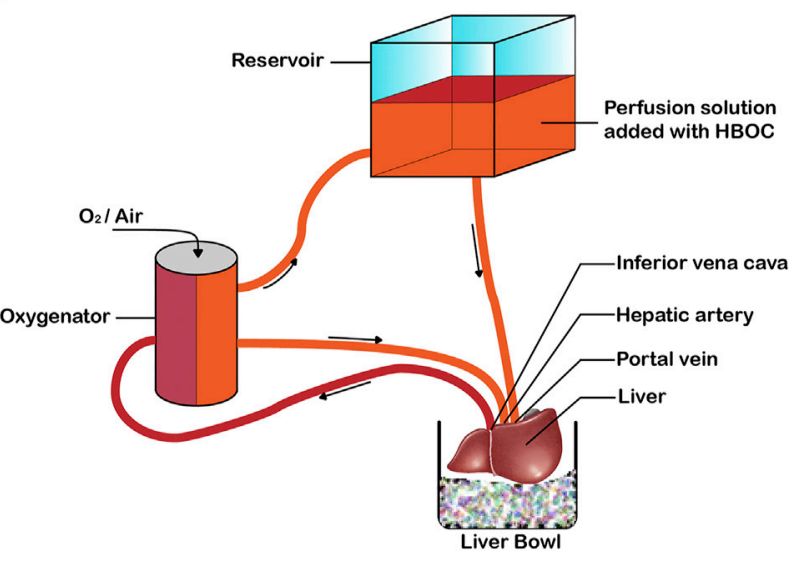

FIGURE 1 | Application of $\mathrm{HBOC}$ in solid organ preservation (e.g., liver). (A) Pre-oxygenation process of preservation solution added with HBOC. (B) Static cold preservation of isolated liver after pre-oxygenation with the preservative solution of added HBOC. (C) Mechanical perfusion of the isolated liver after pre-oxygenation with the preservation fluid of added HBOC.

ketoglutarate (HTK) solutions (Warnecke et al., 2002; Yoshida et al., 2002). The current mainstream organ preservation protocol is still to preserve organs in a standard conventional solution at $4^{\circ} \mathrm{C}$. The purpose of preserving grafts in standard preservation solutions is to slow down the metabolic rate of tissue cells, 


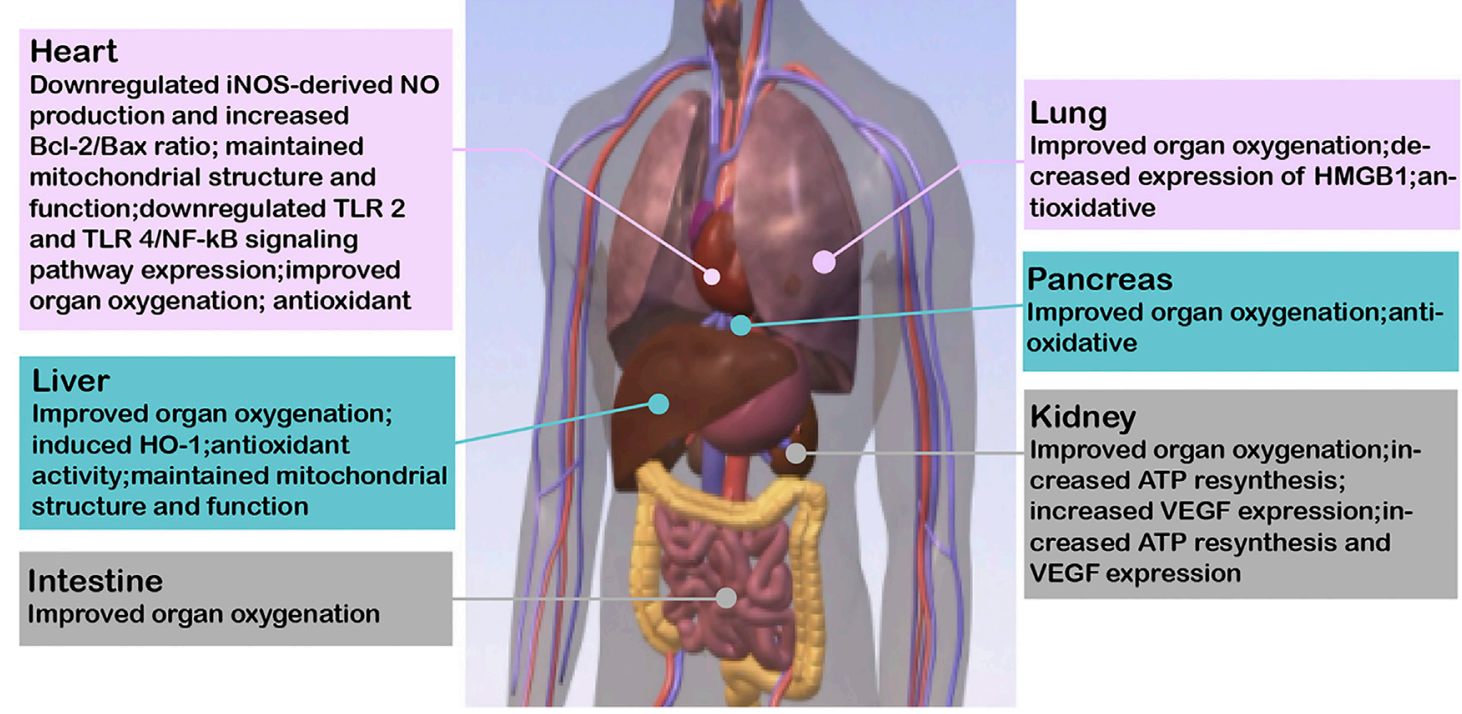

FIGURE 2 | Relevant mechanisms where HBOCs are beneficial to the preservation of solid organs. iNOS, inducible nitric oxide synthase; NO, nitric oxide; Bcl-2, B-cell lymphoma-2; TLR, toll-like receptor; NF-kB, nuclear factor-k-gene binding; HO-1, heme oxygenase-1; ATP, adenosine triphosphate; VEGF, vascular endothelial growth factor.

maintain the balance of substances inside and outside the cells, and reduce cell swelling (Gao et al., 2015; Tian et al., 2009). To further improve graft quality and recipient prognosis, several studies have reported the addition of sugars (Kobayashi et al., 1991), calcium ion antagonists (Ingemansson et al., 1996), potassium channel openers (Han et al., 2009), and plateletactivating factor antagonists (Kim et al., 2000) to the standard preservation solutions. However, the addition of these substances did not improve oxygenation of the tissues. The most fundamental cause of graft injury after removal from the donor is caused by ischemia and hypoxia. The conventional preservation solution with the addition of HBOCs is preoxygenated for a period of time, and the oxygenated solution will be used for static cold preservation and/or mechanical perfusion of the organ (Figure 1A). HBOCs exhibit good compatibility with traditional preservation solutions, and there is no need to modify the characteristics and protocols of organ preservation. Conventional preservation solution with HBOC added performs static cold preservation of the donor organ during the cold ischemic time (time from the perfusion of the organ with the solution to its removal from the cold storage for implantation) (Nakamura et al., 2020), a process that not only reduces cellular energy metabolism but also provides oxygen to meet organ oxygen demand (Figure 1B). The preservation solution containing HBOCs reduces ischemic and hypoxic damage to isolated organs, prolongs the preservation time of organs to meet long-distance transfer, and improves the utilization of organs to some extent. With the advancement of preservation technology and machines that simulate clinical reperfusion, isolated organs can be repaired and evaluated using the MP (machine perfusion) platform after long-distance cold preservation and transportation, which is especially beneficial for the use of marginal organs (Grundmann et al., 1977). Conventional preservation solutions with the addition of HBOCs are combined with different types of MP (hypothermic machine perfusion (HMP), SNMP, NMP, DHOPE [(dual) hypothermic oxygenated machine perfusion-controlled oxygenation rewarming (COR)-NMP]) for the repair and evaluation of marginal organs, which can improve the quality of marginal organs and patient prognosis, expanding to some extent the donor pool (Figure 1C). MP may be used as a platform for the dynamic circulation of isolated organs during preservation, and it induces a more even distribution of HBOCs in blood vessels, promotes the transport and diffusion of oxygen in the microcirculation, and improves the quality of the graft and the prognosis of the recipient (Fontes et al., 2015). Thus, the application of preservation solutions with HBOCs is similar to that of conventional preservation solutions and can be used directly for static cold preservation and mechanical perfusion of isolated organs without significant changes in protocol or equipment (Ali et al., 2020). HBOCs, as a class of oxygen carriers close to physiological conditions, can be used in various types of solid organs with multiple protocols for preservation. HBOCs are safe and feasible oxygen carriers that may be used for the preservation of organs in vitro (Figure 2). The application of HBOCs in different solid organs is discussed in detail below (Table 2).

\section{Heart}

Compared to other solid organs, the clinically isolated heart has a relatively short safe cold storage time (up to $4-6 \mathrm{~h}$ ). The heart is an aerobic organ that relies on a continuous supply of oxygen to produce high-energy phosphate ATP for mechanical functions. The supply of oxygen via HBOCs supports the resynthesis of ATP 
TABLE 2 | Studies evaluating the impact of HBOCs in solid organ transplantation.

\begin{tabular}{|c|c|c|c|c|c|c|c|c|c|}
\hline $\begin{array}{l}\text { Author } \\
\text { and year }\end{array}$ & $\begin{array}{l}\text { Oxygen } \\
\text { carrier }\end{array}$ & $\begin{array}{l}\text { Evaluated } \\
\text { solution }\end{array}$ & $\begin{array}{l}\text { Concen- } \\
\text { tration }\end{array}$ & $\begin{array}{l}\text { Oxyge- } \\
\text { nation }\end{array}$ & $\begin{array}{l}\text { Experimental } \\
\text { proposal }\end{array}$ & Species & Results & $\begin{array}{l}\text { Author } \\
\text { conclusion }\end{array}$ & Limitation/toxicity \\
\hline \multicolumn{10}{|l|}{ Heart } \\
\hline $\begin{array}{l}\text { Li et al. (2009) FREE } \\
\text { RADICAL BIO MED } \\
2009\end{array}$ & PolyPHb & STS & $5 \mathrm{~g} / \mathrm{L}$ & $\begin{array}{l}\text { Equilibrated } \\
\text { with } 95 \% \mathrm{O}_{2} \\
\text { and } 5 \% \mathrm{CO}_{2} \\
\text { at } 37^{\circ} \mathrm{C} \text { for } \\
30 \mathrm{~min}\end{array}$ & $\begin{array}{l}\text { Baseline (30 min; } \\
\text { KHB); HS ( } 8 \text { h; } 5 \mathrm{~g} \\
\mathrm{Hb} / \mathrm{dL} \text { PolyPHb + } \\
\text { STS or blood or } \\
\text { STS); } 2 \text { h KHB NMP }\end{array}$ & $\begin{array}{l}\text { Sprague-Dawley } \\
\text { rats }\end{array}$ & $\begin{array}{l}\text { PolyPHb improved } \\
\text { heart contraction, } \\
\text { decreased infarction } \\
\text { size and myocardial } \\
\text { apoptosis, and } \\
\text { maintained redox } \\
\text { homeostasis }\end{array}$ & $\begin{array}{l}\text { PolyPHb } \\
\text { downregulated } \\
\text { iNOS-derived NO } \\
\text { production and } \\
\text { increased Bcl-2/Bax } \\
\text { ratio; preserved } \\
\text { mitochondrial } \\
\text { function; attenuated } \\
\text { NO-mediated } \\
\text { myocardial } \\
\text { apoptosis; and } \\
\text { restored the nitroso- } \\
\text { redox balance }\end{array}$ & N \\
\hline $\begin{array}{l}\text { Li et al. (2010) FREE } \\
\text { RADICAL BIO MED } \\
2010\end{array}$ & PolyPHb & STS & $0.1 \mathrm{~g} / \mathrm{dl}$ & $\begin{array}{l}\text { Equilibrated } \\
\text { with } 95 \% \mathrm{O}_{2} \\
\text { and } 5 \% \mathrm{CO}_{2} \\
\text { at } 37^{\circ} \mathrm{C} \text { for } \\
30 \mathrm{~min}\end{array}$ & $\begin{array}{l}\text { Sham (150 min } \\
\text { perfusion; KHB) WI } \\
\text { ( } 30 \text { min baseline } \\
\text { KHB; } 35 \text { min STS WI; } \\
2 \text { h KHB NMP); WI + } \\
\text { HBOC ( } 30 \text { min } \\
\text { baseline KHB; } \\
35 \text { min STS + HBOC } \\
\text { WI; } 2 \text { h KHB } \\
\text { reperfusion); HS } \\
\text { (30 min baseline } \\
\text { KHB; } 8 \text { h STS; } 2 \text { h } \\
\text { KHB NMP); HS + } \\
\text { HBOC (30 min } \\
\text { baseline KHB; } 8 \text { h } \\
\text { STS + HBOC; } 2 \text { h } \\
\text { KHB NMP) }\end{array}$ & $\begin{array}{l}\text { Sprague-Dawley } \\
\text { rats }\end{array}$ & $\begin{array}{l}\text { PolyPHb improved } \\
\text { cardiac function; } \\
\text { decreased } \\
\text { myocardial } \\
\text { infarction, necrosis, } \\
\text { and apoptosis; } \\
\text { elevated } \\
\text { mitochondrial } \\
\text { function; and did not } \\
\text { change the } \\
\text { mitochondrial } \\
\text { structure }\end{array}$ & $\begin{array}{l}\text { Attenuated } \\
\text { mitochondrial } \\
\text { oxidative damage; } \\
\text { preserved } \\
\text { mitochondrial } E_{\mathrm{h}} \text { and } \\
\text { SOD activity; } \\
\text { preserved } \\
\text { mitochondrial } \\
\text { function; inhibited } \\
\text { ROS burst; and } \\
\text { depressed } \\
\text { cytochrome C } \\
\text { translocation }\end{array}$ & $\mathrm{N}$ \\
\hline $\begin{array}{l}\text { Wei et al. (2011) } \\
\text { Cardiology } 2011\end{array}$ & PolyPHb & HTKs & $0.3 \mathrm{~g} / \mathrm{dl}$ & $\begin{array}{l}\text { Equilibrated } \\
\text { with } 100 \% \mathrm{O}_{2} \\
\text { at } 37^{\circ} \mathrm{C} \text { for } \\
5 \mathrm{~min}\end{array}$ & $\begin{array}{l}\text { Sham (150 min KHB } \\
\text { perfusion) HTK9 } \\
\text { (30 min baseline } \\
\text { KHB; } 9 \text { h HTKs HS; } \\
2 \text { h KHB NMP); } \\
\text { HBOC9 ( } 30 \text { min } \\
\text { baseline KHB; } 9 \text { h } \\
\text { HTKs + HBOC HS; } \\
2 \text { h KHB NMP); } \\
\text { HTK14 ( } 30 \text { min } \\
\text { baseline KHB; } 14 \text { h } \\
\text { HTKs HS; } 2 \text { h KHB } \\
\text { NMP); HBOC14 } \\
\text { (30 min baseline } \\
\text { KHB; } 14 \text { h HTKs + } \\
\text { HBOC HS; } 2 \text { h } \\
\text { KHB NMP) }\end{array}$ & $\begin{array}{l}\text { Sprague-Dawley } \\
\text { rats }\end{array}$ & $\begin{array}{l}\text { HBOCs improved } \\
\text { heart contraction } \\
\text { and decreased } \\
\text { infarct size, } \\
\text { necrosis, and } \\
\text { apoptosis; reduced } \\
\text { expression of Toll- } \\
\text { like receptor } 2 \text { (TLR } \\
\text { 2), TLR 4, TNF- a, } \\
\text { IL-1 } \beta \text {, and nuclear } \\
\text { factor-kB activation }\end{array}$ & $\begin{array}{l}\text { Downregulated TLR } \\
2 \text { and TLR 4/NF-kB } \\
\text { signaling pathway } \\
\text { expression }\end{array}$ & $\begin{array}{l}\text { Inhibitors of TLR } 2 \text { and TLR } 4 \\
\text { were not used; did not } \\
\text { research the relationship } \\
\text { between different doses of } \\
\text { PolyPHb and TLR } 2 \text { and TLR } \\
\text { 4/NF-kB signaling pathway } \\
\text { expression } \\
\text { ontinued on following page) }\end{array}$ \\
\hline
\end{tabular}


TABLE 2 | (Continued) Studies evaluating the impact of HBOCs in solid organ transplantation.

\begin{tabular}{|c|c|c|c|c|c|c|c|c|c|}
\hline $\begin{array}{l}\text { Author } \\
\text { and year }\end{array}$ & $\begin{array}{l}\text { Oxygen } \\
\text { carrier }\end{array}$ & $\begin{array}{l}\text { Evaluated } \\
\text { solution }\end{array}$ & $\begin{array}{l}\text { Concen- } \\
\text { tration }\end{array}$ & $\begin{array}{l}\text { Oxyge- } \\
\text { nation }\end{array}$ & $\begin{array}{l}\text { Experimental } \\
\text { proposal }\end{array}$ & Species & Results & $\begin{array}{l}\text { Author } \\
\text { conclusion }\end{array}$ & Limitation/toxicity \\
\hline $\begin{array}{l}\text { Teh et al. (2017) } \\
\text { ARTIF CELL } \\
\text { NANOMED B } 2017\end{array}$ & M101 & Celsior & $1 \mathrm{~g} / \mathrm{L}$ & $\begin{array}{l}\text { Equilibrated } \\
\text { with } 95 \% \mathrm{O}_{2} \\
\text { and } 5 \% \mathrm{CO}_{2}\end{array}$ & $\begin{array}{l}4-8^{\circ} \mathrm{C} \text { cold storage } \\
8 \mathrm{~h}(\text { Celsior; Celsior + } \\
\mathrm{M} 101) ; \mathrm{KHB} \\
60 \text { min NMP }\end{array}$ & Wistar rats & $\begin{array}{l}\text { Coronary flow was } \\
\text { significantly higher in } \\
\text { M101 group; } \\
\text { improved } \\
\text { postischemic } \\
\text { recovery of heart } \\
\text { function }\end{array}$ & $\begin{array}{l}\text { Improved organ } \\
\text { oxygenation }\end{array}$ & $\mathrm{N}$ \\
\hline $\begin{array}{l}\text { You et al. (2011) } \\
\text { ARTIF CELL BLOOD } \\
\text { SUB } 2011\end{array}$ & PolyPHb & $\mathrm{KHB}$ & $0.1 \mathrm{~g} / \mathrm{dl}$ & $\begin{array}{l}\text { Equilibrated } \\
\text { with } 95 \% \mathrm{O}_{2} \\
\text { and } 5 \% \mathrm{CO}_{2} \\
\text { for } 10 \mathrm{~min}\end{array}$ & $\begin{array}{l}\text { Sham (perfused by } \\
\text { KHB } 2 \text { h) Control } \\
\text { ( } 40 \text { min baseline; } \\
45 \text { min Wl; } 2 \text { h NMP) } \\
\text { Ischemic } \\
\text { preconditioning } \\
\text { (10 min baseline; } \\
\text { three 5-min ischemia } \\
\text { and } 5 \text {-min KHB } \\
\text { perfusion; } 45 \text { min Wl; } \\
2 \text { h NMP) deoxy- } \\
\text { HBOCs (10 min } \\
\text { baseline; three 5-min } \\
\text { deoxygenated } \\
\text { HBOC perfusion and } \\
5 \text {-min KHB } \\
\text { perfusion; } 45 \text { min W; } \\
2 \mathrm{~h} \text { NMP) }\end{array}$ & $\begin{array}{l}\text { Sprague-Dawley } \\
\text { rats }\end{array}$ & $\begin{array}{l}\text { Deoxygenated } \\
\text { HBOC pretreatment } \\
\text { and ischemic } \\
\text { preconditioning } \\
\text { both equally } \\
\text { improved the } \\
\text { recovery of cardiac } \\
\text { function and } \\
\text { reduced the cardiac } \\
\text { enzyme release and } \\
\text { myocardial } \\
\text { histopathology }\end{array}$ & $\begin{array}{l}\text { Improved organ } \\
\text { oxygenation }\end{array}$ & $\begin{array}{l}\text { Ischemic preconditioning } \\
\text { cannot block the aorta to } \\
\text { induce cardiac ischemia; } \\
\text { results cannot explain the } \\
\text { cardioprotective mechanism }\end{array}$ \\
\hline $\begin{array}{l}\text { White et al. (2015) J } \\
\text { HEART LUNG } \\
\text { TRANSPL } 2015\end{array}$ & HBOC-201 & $\begin{array}{l}\text { STEEN } \\
\text { Solution }\end{array}$ & $40 \mathrm{~g} / \mathrm{L}$ & $\mathrm{N}$ & $\begin{array}{l}\text { RBC group (RBC + } \\
\text { normal saline NMP } \\
6 \text { h); RBC + plasma } \\
\text { group (whole blood } \\
\text { NMP } 6 \text { h); HBOC } \\
\text { group (HBOC-201 } \\
\text { NMP } 6 \text { h); HBOC + } \\
\text { plasma group } \\
\text { (HBOC-201 + } \\
\text { plasma NMP } 6 \text { h) }\end{array}$ & Domestic pigs & $\begin{array}{l}\text { Whole blood-based } \\
\text { perfusate minimized } \\
\text { injury and provided } \\
\text { superior } \\
\text { preservation of } \\
\text { myocardial function } \\
\text { during NMP }\end{array}$ & $\begin{array}{l}\text { HBOCs promoted } \\
\text { spontaneous } \\
\text { oxidation and ROS; } \\
\text { HBOCs increased } \\
\text { the proportion of } \\
\text { methemoglobin and } \\
\text { CVR; HBOCs } \\
\text { increased troponin I } \\
\text { levels and histologic } \\
\text { myocardial injury } \\
\text { scores }\end{array}$ & $\begin{array}{l}\text { Did not directly quantify } \\
\text { methemoglobin; did not } \\
\text { directly test and confirm the } \\
\text { efficacy of the filter in } \\
\text { reducing circulating } \\
\text { leukocytes. Methemoglobin } \\
\text { mediated oxidative damage } \\
\text { of endothelial cells }\end{array}$ \\
\hline \multicolumn{10}{|l|}{ Lung } \\
\hline $\begin{array}{l}\text { Glorion et al. (2018) } \\
\text { ARTIF CELL } \\
\text { NANOMED B } 2017\end{array}$ & M101 & $\begin{array}{l}\text { PerfadexV R } \\
\text { (Xvivo, } \\
\text { Sweden) }\end{array}$ & $2 \mathrm{~g} / \mathrm{L}$ & $\mathrm{N}$ & $\begin{array}{l}\text { Sham (similar to the } \\
\text { recipient animals but } \\
\text { without undergoing } \\
\text { lung transplantation); } \\
\text { Perfadex }\left(4-6^{\circ} \mathrm{C} 24 \mathrm{~h}\right. \\
\text { cold storage); } \mathrm{M} 101 \\
+ \text { Perfadex (4-6 } 6^{\circ} \mathrm{C} \\
24 \mathrm{~h} \text { cold storage) }\end{array}$ & White pigs & $\begin{array}{l}\text { M101 reduced graft } \\
\text { vascular resistance } \\
\text { and increased the } \\
\text { graft oxygenation } \\
\text { ratio }\end{array}$ & $\begin{array}{l}\text { Improved organ } \\
\text { oxygenation and } \\
\text { decreased } \\
\text { expression of } \\
\text { HMGB1 }\end{array}$ & $N$ \\
\hline $\begin{array}{l}\text { Ali et al. (2020) J } \\
\text { HEART LUNG } \\
\text { TRANSPL } 2020\end{array}$ & $\begin{array}{l}\text { M101 static } \\
\text { cold storage } \\
\text { (SCS) ex vivo }\end{array}$ & $\begin{array}{l}\text { Low- } \\
\text { potassium }\end{array}$ & $1 \mathrm{~g} / \mathrm{L}$ & $N$ & $\begin{array}{l}\text { LPD (SCS } 36 \mathrm{~h}+ \\
\text { EVLP } 12 \text { h); M101 + }\end{array}$ & Yorkshire pigs & $\begin{array}{l}\text { M101 provided } \\
\text { better physiologic } \\
\text { parameters and }\end{array}$ & $\begin{array}{l}\text { Improved organ } \\
\text { oxygenation; }\end{array}$ & $\begin{array}{l}\text { Did not cause donor-related } \\
\text { injuries before cold } \\
\text { preservation; EVLP } \\
\text { Continued on following page) }\end{array}$ \\
\hline
\end{tabular}


TABLE 2 | (Continued) Studies evaluating the impact of HBOCs in solid organ transplantation.

\begin{tabular}{|c|c|c|c|c|c|c|c|c|c|}
\hline $\begin{array}{l}\text { Author } \\
\text { and year }\end{array}$ & $\begin{array}{l}\text { Oxygen } \\
\text { carrier }\end{array}$ & $\begin{array}{l}\text { Evaluated } \\
\text { solution }\end{array}$ & $\begin{array}{l}\text { Concen- } \\
\text { tration }\end{array}$ & $\begin{array}{l}\text { Oxyge- } \\
\text { nation }\end{array}$ & $\begin{array}{l}\text { Experimental } \\
\text { proposal }\end{array}$ & Species & Results & $\begin{array}{l}\text { Author } \\
\text { conclusion }\end{array}$ & Limitation/toxicity \\
\hline & $\begin{array}{l}\text { lung perfusion } \\
\text { (EVLP) }\end{array}$ & $\begin{array}{l}\text { dextran } \\
\text { solution (LPD) }\end{array}$ & & & $\begin{array}{l}\text { LPD (SCS } 36 \mathrm{~h}+ \\
\text { EVLP } 12 \mathrm{~h})\end{array}$ & & $\begin{array}{l}\text { oxygenation; } \\
\text { reduced edema } \\
\text { formation and } \\
\text { apoptotic cell death; } \\
\text { improved tight } \\
\text { junction } \\
\text { preservation; } \\
\text { reduced the level of } \\
\text { IL-6 in recipient } \\
\text { plasma }\end{array}$ & $\begin{array}{l}\text { antioxidative } \\
\text { properties }\end{array}$ & $\begin{array}{l}\text { minimized the physiologic } \\
\text { differences between the } \\
\text { experimental groups; the } \\
\text { optimal dose of the EOC and } \\
\text { maximal tolerable ischemic } \\
\text { times were not investigated }\end{array}$ \\
\hline \multicolumn{10}{|c|}{ 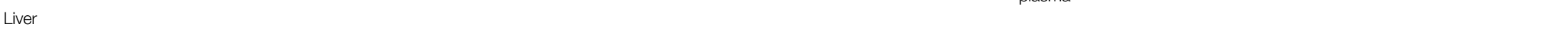 } \\
\hline $\begin{array}{l}\text { Topp et al. (2008) J } \\
\text { SURG RES } 2008\end{array}$ & $\mathrm{HbG} 200$ & $\mathrm{KH}-\mathrm{Sol}$ & $3.3 \mathrm{~g} / \mathrm{dl}$ & $\begin{array}{l}\text { Equilibrated } \\
\text { with } 95 \% \mathrm{O}_{2} \\
\text { and } 5 \% \mathrm{CO}_{2}\end{array}$ & $\begin{array}{l}\text { KH-Sol (EC cold } \\
\text { storage } 24 \mathrm{~h} \text {; rat } \\
\text { blood + KH-Sol IPRL } \\
180 \text { min); KH-Sol + } \\
\text { HbG (EC cold } \\
\text { storage } 24 \mathrm{~h} \text {; rat } \\
\text { blood + KH-Sol + } \\
\text { HbG IPRL } 180 \text { min); } \\
\text { KH-Sol.+6\% HES } \\
\text { (EC cold storage } \\
24 \text { h; rat blood and + } \\
\text { KH-Sol + 6\% HES } \\
\text { IPRL } 180 \text { min); KH- } \\
\text { Sol + HbG + SnPP } \\
\text { pretreated (EC cold } \\
\text { storage } 24 \text { h; rat } \\
\text { blood and + KH-Sol } \\
+ \text { HbG + SnPP IPRL } \\
180 \text { min) }\end{array}$ & Wistar rat & $\begin{array}{l}\text { HBOCs increased } \\
\text { HO-1 expression } \\
\text { and activity during } \\
\text { reperfusion, which } \\
\text { could be abolished } \\
\text { by tin } \\
\text { protoporphyrin IX } \\
\text { application }\end{array}$ & $\begin{array}{l}\text { Improved organ } \\
\text { oxygenation and } \\
\text { induced } \mathrm{HO}-1 \text {; the } \\
\text { antioxidant and anti- } \\
\text { inflammatory } \\
\text { properties of HO-1 }\end{array}$ & $\begin{array}{l}\text { Endothelin-1-mediated } \\
\text { vasoconstriction }\end{array}$ \\
\hline $\begin{array}{l}\text { Fontes et al. (2015) } \\
\text { AM J TRANSPLANT } \\
2015\end{array}$ & HBOC-201 & UW & $3.5 \mathrm{~g} / \mathrm{dl}$ & $\begin{array}{l}\text { Oxygenated to } \\
\mathrm{SaO}_{2}>95 \% \\
\text { and } \mathrm{paO}_{2}> \\
400 \mathrm{mmHg}\end{array}$ & $\begin{array}{l}\text { CSP (cold storage } \\
9 \mathrm{~h}) \mathrm{MP}\left(21^{\circ} \mathrm{C}, \mathrm{UW}+\right. \\
\mathrm{HBOC} 9 \mathrm{~h})\end{array}$ & Landrace pigs & $\begin{array}{l}\text { MP/HBOC system } \\
\text { had higher survival } \\
\text { and superior graft } \\
\text { function; oxygen } \\
\text { delivered by the liver } \\
\text { was } 8 \text { times that of } \\
\text { oxygen intake; } \\
\text { significantly } \\
\text { improved liver } \\
\text { preservation }\end{array}$ & $\begin{array}{l}\text { Improved organ } \\
\text { oxygenation }\end{array}$ & $N$ \\
\hline $\begin{array}{l}\text { Matton et al. (2018) } \\
\text { LIVER TRANSPLANT } \\
2018\end{array}$ & HBOC-201 & $\begin{array}{l}20 \% \text { Human } \\
\text { albumin or } \\
4 \% \text { gelofusine }\end{array}$ & $18.3 \mathrm{~g} / \mathrm{L}$ & $\begin{array}{l}\text { Equilibrated } \\
\text { with } 95 \% \mathrm{O}_{2} \\
\text { and } 5 \% \mathrm{CO}_{2}\end{array}$ & $\begin{array}{l}\text { RBC + FFP }(6 \text { h } \\
\text { NMP); HBOC- } 201+ \\
\text { FFP ( } 6 \text { h NMP); } \\
\text { HBOC-201 + } \\
\text { gelofusine ( } 6 \text { h NMP) }\end{array}$ & Human & $\begin{array}{l}\text { HBOC-201 had } \\
\text { significantly higher } \\
\text { hepatic adenosine } \\
\text { triphosphate } \\
\text { content, cumulative } \\
\text { bile production, and } \\
\text { portal and arterial } \\
\text { flows }\end{array}$ & $\begin{array}{l}\text { Improved organ } \\
\text { oxygenation }\end{array}$ & $\begin{array}{l}\text { Relatively small sample sizes; } \\
\text { lack of transplant validation; } \\
\text { study grouping was } \\
\text { performed consecutively } \\
\text { rather than after } \\
\text { randomization }\end{array}$ \\
\hline
\end{tabular}


TABLE 2 | (Continued) Studies evaluating the impact of HBOCs in solid organ transplantation.

\begin{tabular}{|c|c|c|c|c|c|c|c|c|c|}
\hline $\begin{array}{l}\text { Author } \\
\text { and year }\end{array}$ & $\begin{array}{l}\text { Oxygen } \\
\text { carrier }\end{array}$ & $\begin{array}{l}\text { Evaluated } \\
\text { solution }\end{array}$ & $\begin{array}{l}\text { Concen- } \\
\text { tration }\end{array}$ & $\begin{array}{l}\text { Oxyge- } \\
\text { nation }\end{array}$ & $\begin{array}{l}\text { Experimental } \\
\text { proposal }\end{array}$ & Species & Results & $\begin{array}{l}\text { Author } \\
\text { conclusion }\end{array}$ & Limitation/toxicity \\
\hline $\begin{array}{l}\text { Laing et al. (2017) } \\
\text { Transplantation } 2017\end{array}$ & HBOC-201 & $\begin{array}{l}\text { Modified } \\
\text { Ringer's } \\
\text { lactate } \\
\text { solution }\end{array}$ & $63.7 \mathrm{~g} / \mathrm{L}$ & $\begin{array}{l}\text { Oxygenated to } \\
\text { arterial } \mathrm{pO}_{2}= \\
20 \mathrm{kPa}\end{array}$ & $\begin{array}{l}\text { HBOCs (Hemopure- } \\
\text { based perfusion fluid } \\
6 \text { h NMP); RBCs } \\
\text { (packed red blood } \\
\text { cell-based fluid } \\
6 \text { h NMP) }\end{array}$ & Human & $\begin{array}{l}\text { HBOC-perfused } \\
\text { livers extracted } \\
\text { more oxygen than } \\
\text { those perfused with } \\
\text { RBCs }\end{array}$ & $\begin{array}{l}\text { Improved organ } \\
\text { oxygenation; } \\
\text { exhibited antioxidant } \\
\text { activity }\end{array}$ & $\begin{array}{l}\text { Livers were not transplanted. } \\
\text { Did not simulate the } \\
\text { reperfusion with whole blood } \\
\text { containing immune cell } \\
\text { populations }\end{array}$ \\
\hline $\begin{array}{l}\text { van Leeuwen et al. } \\
\text { (2019). Ann Surg } 2019\end{array}$ & HBOC-201 & UW & $\mathrm{N}$ & $\begin{array}{l}\text { DHOPE: } \\
\text { oxygenated to } \\
\mathrm{PaO}_{2}> \\
80 \mathrm{kPa}\end{array}$ & $\begin{array}{l}1 \mathrm{~h} \text { DHOPE; } 1 \mathrm{~h} \\
\text { COR; } 150 \text { min NMP }\end{array}$ & Human & $\begin{array}{l}69 \% \text { of livers that } \\
\text { met all viability } \\
\text { criteria were } \\
\text { successfully } \\
\text { transplanted; } 100 \% \\
\text { graft survival at } 3 \\
\text { and } 6 \text { months; } \\
\text { DHOPE-COR-NMP } \\
\text { increased the } \\
\text { number of } \\
\text { deceased donor } \\
\text { liver transplants } \\
\text { by 20\% }\end{array}$ & $\begin{array}{l}\text { Improved organ } \\
\text { oxygenation }\end{array}$ & $\begin{array}{l}\text { Lack of randomization; livers } \\
\text { were not transplanted based } \\
\text { on a low bile pH during NMP, } \\
\text { and therefore have no } \\
\text { follow-up }\end{array}$ \\
\hline $\begin{array}{l}\text { Shonaka et al. (2019) } \\
\text { PLoS One } 2019\end{array}$ & $\mathrm{HbV}$ & UW & $\mathrm{N}$ & $\mathrm{N}$ & $\begin{array}{l}\text { CS (WIT } 1 \text { h,CS } 4 \text { h); } \\
\text { HMP (WIT } 1 \mathrm{~h}, \text { HMP } \\
4 \text { h); SNMP (WIT } 1 \mathrm{~h} \text {, } \\
\text { SNMP } 4 \text { h); SNMP + } \\
\text { HbV (WIT } 1 \text { h, SNMP } \\
\text { + HbV } 4 \text { h) }\end{array}$ & $\begin{array}{l}\text { Female cross-bred } \\
\text { Large-Yorkshire, } \\
\text { Landrace, and } \\
\text { Duroc pigs }\end{array}$ & $\begin{array}{l}\text { SNMP + HbV } \\
\text { solution can reduce } \\
\text { the reperfusion } \\
\text { injury of DCD donor } \\
\text { liver. The } \\
\text { mitochondria, pH } \\
\text { and lactate levels of } \\
\text { the SNMP + HbV } \\
\text { group were well } \\
\text { maintained in } \\
\text { comparison to the } \\
\text { CS, HMP, and } \\
\text { SNMP groups }\end{array}$ & $\begin{array}{l}\text { Increased } \\
\text { oxygenation and } \\
\text { oxygen } \\
\text { consumption, } \\
\text { maintained } \\
\text { mitochondrial } \\
\text { structure and } \\
\text { function, and had a } \\
\text { protective effect on } \\
\text { metabolic acidosis }\end{array}$ & $\begin{array}{l}\text { Transplantation was not } \\
\text { performed, and these results } \\
\text { are not sufficient for } \\
\text { predicting the effects of } \\
\text { SNMP/HbV in a living body }\end{array}$ \\
\hline $\begin{array}{l}\text { Alix et al. (2020) } \\
\text { JHEP Rep } 2020\end{array}$ & M101 & UW & $1 \mathrm{~g} / \mathrm{L}$ & $\mathrm{N}$ & $\begin{array}{l}\text { SCS (cold storage, } \\
\text { UW } 6 \text { h); SCS + } \\
\text { M101 (cold storage, } \\
\text { UW+1 g/L M101 } \\
6 \text { h); HOPE (HOPE, } \\
\text { UW+1 g/L M101) }\end{array}$ & $\begin{array}{l}\text { White/Landrace } x \\
\text { Piétrain pigs }\end{array}$ & $\begin{array}{l}\text { M101 effectively } \\
\text { oxygenated liver } \\
\text { grafts during } \\
\text { preservation; } \\
\text { prevented } \\
\text { posttransplant injury } \\
\text { without reaching the } \\
\text { level of HOPE }\end{array}$ & $\begin{array}{l}\text { Improved organ } \\
\text { oxygenation and } \\
\text { preserved ATP } \\
\text { synthase activity }\end{array}$ & $\begin{array}{l}\text { Two different solutions were } \\
\text { used in the SCS and HOPE } \\
\text { groups; no medicoeconomic } \\
\text { data available regarding the } \\
\text { potential benefit of M101 in } \\
\text { liver transplantation }\end{array}$ \\
\hline $\begin{array}{l}\text { Boteon et al. (2019) } \\
\text { PLoS One } 2019\end{array}$ & HBOC-201 & UW & $\mathrm{N}$ & $\begin{array}{l}\text { Equilibrated } \\
\text { with } 30 \% \mathrm{O}_{2} \\
1-2 \mathrm{~L} / \mathrm{min}\end{array}$ & $\begin{array}{l}\text { HOPE + NMP (UW + } \\
\text { HOPE } 2 \text { h, UW + } \\
\text { HBOC-201 + NMP } \\
4 \text { h); cold-to-warm } \\
\text { group (UW + HBOC- } \\
201 \text { + HOPE, } \\
\text { gradually rewarmed, }\end{array}$ & Human & $\begin{array}{l}\text { Both HMP + NMP } \\
\text { and cold-to-warm } \\
\text { mitigated the } \\
\text { oxidative-mediated } \\
\text { tissue injury and } \\
\text { enhanced hepatic } \\
\text { energy stores; HMP } \\
\text { + NMP simplified }\end{array}$ & $\begin{array}{l}\text { Improved organ } \\
\text { oxygenation and } \\
\text { enhanced ATP } \\
\text { synthesis }\end{array}$ & $\begin{array}{l}\text { Relatively small sample sizes; } \\
\text { organs were not } \\
\text { transplanted; replacement of } \\
\text { the perfusate may reduce } \\
\text { toxic metabolites; unable to } \\
\text { evaluate transaminases or } \\
\text { other markers of oxidative } \\
\text { stress in the perfusate; } \\
\text { Continued on following page) }\end{array}$ \\
\hline
\end{tabular}


TABLE 2 | (Continued) Studies evaluating the impact of HBOCs in solid organ transplantation.

\begin{tabular}{|c|c|c|c|c|c|c|c|c|c|}
\hline $\begin{array}{l}\text { Author } \\
\text { and year }\end{array}$ & $\begin{array}{l}\text { Oxygen } \\
\text { carrier }\end{array}$ & $\begin{array}{l}\text { Evaluated } \\
\text { solution }\end{array}$ & $\begin{array}{l}\text { Concen- } \\
\text { tration }\end{array}$ & $\begin{array}{l}\text { Oxyge- } \\
\text { nation }\end{array}$ & $\begin{array}{l}\text { Experimental } \\
\text { proposal }\end{array}$ & Species & Results & $\begin{array}{l}\text { Author } \\
\text { conclusion }\end{array}$ & Limitation/toxicity \\
\hline & & & & & $\begin{array}{l}\text { NMP for viability } \\
\text { assessment, } 6 \text { h) }\end{array}$ & & $\begin{array}{l}\text { the logistics of this } \\
\text { combination and } \\
\text { was conducive to } \\
\text { clinical applicability }\end{array}$ & & $\begin{array}{l}\text { absolute values and } \\
\text { proportional ATP increase } \\
\text { were lower than those in } \\
\text { other studies performing } \\
\text { HOPE; lack of further data on } \\
\text { the histology of the bile duct }\end{array}$ \\
\hline $\begin{array}{l}\text { de Vries et al. (2019) } \\
\text { Am J Transplant. } 2019\end{array}$ & HBOC-201 & UW & $\mathrm{N}$ & $\begin{array}{l}\text { 3-Month graft } \\
\text { survival } \\
\text { was 100\% }\end{array}$ & $\begin{array}{l}\text { DHOPE, } 10^{\circ} \mathrm{C} 1 \mathrm{~h} \text {; } \\
\text { COR, the } \\
\text { temperature was } \\
\text { gradually increased } \\
\text { approximately } 1^{\circ} \mathrm{C} \\
\text { per } 2 \text { min to } 37^{\circ} \mathrm{C} \text {; } \\
\text { NMP, } 37^{\circ} \mathrm{C} 1 \mathrm{~h}\end{array}$ & Human & $\begin{array}{l}\text { The 3-month graft } \\
\text { survival rate } \\
\text { was 100\% }\end{array}$ & $\begin{array}{l}\text { Improved organ } \\
\text { oxygenation }\end{array}$ & $\begin{array}{l}\text { Relatively small sample sizes } \\
\text { and lack of a control group; } \\
\text { did not draw conclusions on } \\
\text { the value of the COR phase; } \\
\text { did not discriminate between } \\
\text { the beneficial effects of } \\
\text { DHOPE, COR, and NMP } \\
\text { separately; HBOCs were } \\
\text { converted to } \\
\text { methemoglobin, especially in } \\
\text { the venous phase }\end{array}$ \\
\hline $\begin{array}{l}\text { Shonaka et al. (2018) } \\
\text { Transplant Proc } 2018\end{array}$ & $\mathrm{HbV}$ & UW & $\begin{array}{l}0.6-0.7 \mathrm{mg} / \\
\mathrm{dl}\end{array}$ & $N$ & $\begin{array}{l}\text { Group } 1 \text { (cold } \\
\text { storage UW } 240 \text { min, } \\
\text { reperfusion with } \\
\text { autologous diluted } \\
\text { blood } 120 \text { min); } \\
\text { Group } 2(\mathrm{SNMP} \\
\left(20-22^{\circ} \mathrm{C} \text { ) UW }\right. \\
240 \text { min, reperfusion } \\
\text { with autologous } \\
\text { diluted blood } \\
120 \text { min); Group } 3 \\
\text { (SNMP HbV and UW } \\
240 \text { min, reperfusion } \\
\text { with autologous } \\
\text { diluted blood } \\
120 \text { min) }\end{array}$ & $\begin{array}{l}\text { Female cross-bred } \\
\text { Large-Yorkshire, } \\
\text { Landrace, and } \\
\text { Duroc pigs }\end{array}$ & $\begin{array}{l}\mathrm{HbV} \text { increased the } \\
\text { oxygen } \\
\text { consumption of the } \\
\text { donor liver during } \\
\text { SNMP }\end{array}$ & $\begin{array}{l}\text { Improved organ } \\
\text { oxygenation }\end{array}$ & $\begin{array}{l}\text { Animal model; experiment on } \\
\text { the transplant model, and } \\
\text { therefore, the result of the } \\
\text { actual transplant is unknown }\end{array}$ \\
\hline \multicolumn{10}{|l|}{ Kidney } \\
\hline $\begin{array}{l}\text { Thuillier et al. (2011), } \\
\text { AM J TRANSPLANT } \\
2011\end{array}$ & M101 & UW or HTK & $1 \mathrm{~g} / \mathrm{L}$ & N & $\begin{array}{l}\text { Controls (sham- } \\
\text { operated); UW (CS } \\
24 \text { h); UW + } 5 \text { g/ } \\
\text { LM101 (CS } 24 \text { h); } \\
\text { HTK (CS } 24 \text { h); HTK } \\
+5 \text { g/L M101 } \\
\text { (CS } 24 \text { h) }\end{array}$ & $\begin{array}{l}\text { Large white } \\
\text { male pig }\end{array}$ & $\begin{array}{l}\text { HBOCs lowered the } \\
\text { peak of serum } \\
\text { creatinine, reduced } \\
\text { kidney inflammation } \\
\text { levels, and } \\
\text { maintained } \\
\text { structural integrity; } \\
\text { improved survival } \\
\text { and function; and } \\
\text { slowed the advance } \\
\text { of interstitial fibrosis }\end{array}$ & $\begin{array}{l}\text { Improved organ } \\
\text { oxygenation and } \\
\text { provided SOD activity }\end{array}$ & $N$ \\
\hline $\begin{array}{l}\text { Mallet et al. (2014) } \\
\text { Artif Organs } 2014\end{array}$ & M101 & UW & $\begin{array}{l}1 \mathrm{~g} / \mathrm{L} ; 2 \mathrm{~g} / \mathrm{L} ; \\
5 \mathrm{~g} / \mathrm{L}\end{array}$ & $N$ & $\begin{array}{l}\text { UW solution (CS } \\
24 \text { h); UW+ } 1 \mathrm{~g} / \mathrm{L} \\
\text { M101 (CS } 24 \text { h); }\end{array}$ & $\begin{array}{l}\text { Large white male } \\
\text { pigs }\end{array}$ & $\begin{array}{l}\text { Cells preserved } \\
\text { better with } \\
\text { HEMO2Life in a }\end{array}$ & $\begin{array}{l}\text { Improved organ } \\
\text { oxygenation }\end{array}$ & ontinued on following page) \\
\hline
\end{tabular}


TABLE 2 | (Continued) Studies evaluating the impact of HBOCs in solid organ transplantation

\begin{tabular}{lllll}
\hline $\begin{array}{l}\text { Author } \\
\text { and year }\end{array}$ & $\begin{array}{l}\text { Oxygen } \\
\text { carrier }\end{array}$ & $\begin{array}{c}\text { Evaluated } \\
\text { solution }\end{array}$ & $\begin{array}{c}\text { Concen- } \\
\text { tration }\end{array}$ & $\begin{array}{c}\text { Oxyge- } \\
\text { nation }\end{array}$
\end{tabular}

$\begin{gathered}\text { Experimental } \\ \text { proposal }\end{gathered}$
UW+ $2 \mathrm{~g} / \mathrm{L} \mathrm{M} 101$
$(\mathrm{CS} 24 \mathrm{~h}) ; \mathrm{UW}+5 \mathrm{~g} / \mathrm{L}$
$\mathrm{M} 101(\mathrm{CS} 24 \mathrm{~h})$

\begin{abstract}
Mahboub et al.
\end{abstract}
HBOC-201

2019

Aburawi et al. (2019) HBOC-201 AM J TRANSPLANT

2019

Kasil et al. (2019) Int. M101 J. Mol. Sci 2019
UW

Williams E

Media

PERF-GEN ${ }^{\circledast}$

preservation

solution

Equilibrated
with $95 \% \mathrm{O}_{2}$
and $5 \% \mathrm{CO}_{2}$,
$\mathrm{SaO}_{2}>97 \%$
Equilibrated
with $95 \% \mathrm{O}_{2}$
and $5 \% \mathrm{CO}_{2}$
N

UW $\left(37^{\circ} \mathrm{C} 30 \mathrm{~min}\right.$, CS Lewis rats

$4^{\circ} \mathrm{C} 120 \mathrm{~min}$

rewarming $90 \mathrm{~min}$

$10-37^{\circ} \mathrm{C}$,

anastomosis $30 \mathrm{~min}$

$21^{\circ} \mathrm{C}$, reperfusion

$37^{\circ} \mathrm{C} 120$ min with

HBOCs + UW)

HBOCs $\left(37^{\circ} \mathrm{C}\right.$

$30 \mathrm{~min}, \mathrm{CS} 4^{\circ} \mathrm{C}$

120 min, rewarming

90 min $10-37^{\circ} \mathrm{C}$ with

HBOCs,

anastomosis $30 \mathrm{~min}$

$21^{\circ} \mathrm{C}$, reperfusion

$37^{\circ} \mathrm{C} 120$ min with

HBOCs)

HBOCs (6 h NMP); Human

PRBCs (6 h NMP)

Group W $(1 \mathrm{~h} \mathrm{Wl}$,

23 h HMP); Group

W- $\mathrm{O}_{2}(1 \mathrm{~h} \mathrm{WI}, 23 \mathrm{~h}$

HMP oxygenated

with $100 \% \mathrm{O}_{2}$ at $1 \mathrm{~L}$

min); Group W-M10

(1 h Wl, $23 \mathrm{~h}$

$\mathrm{HMP}+2 \mathrm{~g} / \mathrm{L}$ M101);

Group W- $\mathrm{O}_{2}+\mathrm{M} 101$

(1 h Wl, 23 h HMP

oxygenated with

$100 \% \mathrm{O}_{2}$ at $1 \mathrm{~L}$

$\mathrm{min}+2 \mathrm{~g} / \mathrm{L}$ M101)

SCS ( 30 min WI, CS Landrace pigs

HTK 8 h) $22^{\circ} \mathrm{C}$ blood

Results

dose-dependent

manner; better

survival, metabolic

activity, and cellular

integrity in vitro;

creatinine and

fibrosis levels similar

to those in

pretransplant

kidneys

ultrafiltrate

improved

HBOCs improved Improved organ

kidney function and oxygenation

production, and

glomerular filtration

rate and sodium

reabsorption
HBOC/NMP system Improved organ was feasible and did oxygenation

not result in inferior

outcomes

compared to

PRBCs

M101 associated Provided intrinsic

with or without SOD activity and

$100 \% \mathrm{O}_{2}$ improved regulated $\mathrm{pO}_{2}$;

kidney recovery and maintained ROS/

late graft outcome anti-ROS balance
Lack of transplantation

validation; did not specifically study the $\mathrm{O}_{2}$ carrying

capacity; Methemoglobin

increased slightly and more

during reperfusion

Relatively small sample sizes; lack of transplantation or a simulated reperfusion component

Improved organ oxygenation:
N

(Continued on following page) 
TABLE 2 | (Continued) Studies evaluating the impact of HBOCs in solid organ transplantation.

\begin{tabular}{|c|c|c|c|c|c|c|c|c|c|}
\hline $\begin{array}{l}\text { Author } \\
\text { and year }\end{array}$ & $\begin{array}{l}\text { Oxygen } \\
\text { carrier }\end{array}$ & $\begin{array}{l}\text { Evaluated } \\
\text { solution }\end{array}$ & $\begin{array}{l}\text { Concen- } \\
\text { tration }\end{array}$ & $\begin{array}{l}\text { Oxyge- } \\
\text { nation }\end{array}$ & $\begin{array}{l}\text { Experimental } \\
\text { proposal }\end{array}$ & Species & Results & $\begin{array}{l}\text { Author } \\
\text { conclusion }\end{array}$ & Limitation/toxicity \\
\hline $\begin{array}{l}\text { TRANSPLANTATION } \\
2019\end{array}$ & & & $\begin{array}{l}\text { PlasmaLyte } \\
\text { solution }(1: 2)\end{array}$ & & $\begin{array}{l}(30 \mathrm{~min} \text { Wl, CS blood } \\
4 \mathrm{~h}, \text { blood SNMP } \\
4 \mathrm{~h}) 22^{\circ} \mathrm{C} \text { HBOCs } \\
(30 \mathrm{~min} \text { WI, CS blood } \\
4 \mathrm{~h}, \mathrm{HBOCs} \\
\text { SNMP } 4 \mathrm{~h})\end{array}$ & & $\begin{array}{l}\text { flow and function } \\
\text { compared with } \\
\text { blood; reduced } \\
\text { acute tubular } \\
\text { necrosis (ATN) } \\
\text { scores and degrees } \\
\text { of TUNEL staining; } \\
\text { and reduced urinary } \\
\text { damage markers } \\
\text { and IL-6 }\end{array}$ & $\begin{array}{l}\text { negative } \\
\text { proinflammatory } \\
\text { effect }\end{array}$ & \\
\hline $\begin{array}{l}\text { Kaminski et al. (2019) } \\
\text { Transpl Int } 2019\end{array}$ & M101 & $\begin{array}{l}\text { UW (CS) } \\
\text { KPS (MP) }\end{array}$ & $\begin{array}{l}1 \mathrm{~g} / \mathrm{L} \text { or } \\
2 \mathrm{~g} / \mathrm{L}\end{array}$ & $\mathrm{N}$ & 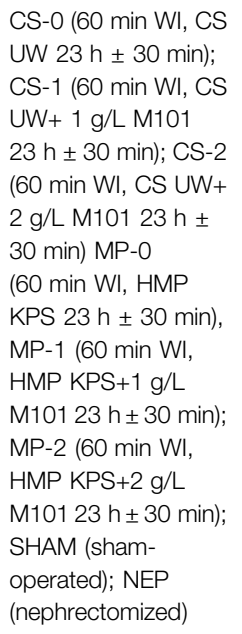 & $\begin{array}{l}\text { Large white male } \\
\text { pigs }\end{array}$ & $\begin{array}{l}\text { Cs arm: } \\
\text { M101 dose- } \\
\text { dependently } \\
\text { improved long-term } \\
\text { function MP arm: } \\
\text { M101 improved } \\
\text { short and long-term } \\
\text { functional outcomes } \\
\text { as well as tissue } \\
\text { integrity }\end{array}$ & $\begin{array}{l}\text { Improved organ } \\
\text { oxygenation and } \\
\text { increased ATP } \\
\text { resynthesis and } \\
\text { VEGF expression }\end{array}$ & $N$ \\
\hline $\begin{array}{l}\text { Le Meur et al. (2020) } \\
\text { Am J Transplant. } 2020\end{array}$ & M101 & UW & $1 \mathrm{~g} / \mathrm{L}$ & $N$ & $\begin{array}{l}\text { M101 (CS or MP); } \\
\text { UW (CS or MP). } \\
\text { M101 added to the } \\
\text { preservation solution } \\
\text { of one of two kidneys } \\
\text { from the same donor }\end{array}$ & Human & $\begin{array}{l}\text { M101 significantly } \\
\text { reduced DGF and } \\
\text { improved renal } \\
\text { function }\end{array}$ & $\begin{array}{l}\text { Improved organ } \\
\text { oxygenation }\end{array}$ & $N$ \\
\hline \multicolumn{10}{|l|}{ Pancreas } \\
\hline $\begin{array}{l}\text { Avila et al. (2006) Am } \\
\text { J Transplant } 2006\end{array}$ & $\begin{array}{l}\text { Polymerized } \\
\text { human } \\
\text { hemoglobin }\end{array}$ & Poly SFH-P & $10 \mathrm{~g} / \mathrm{dl}$ & $\begin{array}{l}\text { Equilibrated } \\
\text { with } 100 \% \mathrm{O}_{2} \\
\text { for } 15 \mathrm{~min}\end{array}$ & $\begin{array}{l}\text { Poly SFH-P ( } 30 \text { min } \\
\text { Wl; } 37^{\circ} \mathrm{C} 18 \text { min } \\
\text { perfusion); Control } \\
\text { (30 min Wl; } 37^{\circ} \mathrm{C} \\
18 \text { min perfusion) }\end{array}$ & Lewis rats & $\begin{array}{l}\text { Poly SFH-P } \\
\text { improved islet } \\
\text { isolation } \\
\text { oxygenation and } \\
\text { preserved } \\
\text { mitochondrial } \\
\text { integrity }\end{array}$ & $\begin{array}{l}\text { Improved organ } \\
\text { oxygenation; } \\
\text { improved islet } \\
\text { viability; and } \\
\text { improved integrity of } \\
\text { both beta and non- } \\
\text { beta cells }\end{array}$ & $\mathrm{N}$ \\
\hline $\begin{array}{l}\text { Espes et al. (2015) } \\
\text { Transplantation } 2015\end{array}$ & PolyHb & $\begin{array}{l}\text { Phosphate } \\
\text { buffer (PB) }\end{array}$ & $\begin{array}{l}108.79 \mathrm{mg} / \\
\mathrm{ml}\end{array}$ & $\mathrm{N}$ & $\begin{array}{l}\text { PolyHb }(0.03 \text { mg/g); } \\
\text { PolyHb }(0.1 \mathrm{mg} / \mathrm{g}) ; \\
\text { PolyHb }(0.3 \mathrm{mg} / \mathrm{g}) ; \\
\text { Recipient: C57Bᄂ } \\
6 \text { nu/nu mice }\end{array}$ & Male C57BL/6 & $\begin{array}{l}\text { PolyHb effectively } \\
\text { bridged the critical } \\
\text { hypoxic phase } \\
\text { immediately after } \\
\text { transplantation, }\end{array}$ & $\begin{array}{l}\text { Improved organ } \\
\text { oxygenation }\end{array}$ & $\begin{array}{l}\text { Macrophages were attracted } \\
\text { by the presence of the } \\
\text { hemoglobin-haptoglobin } \\
\text { complex in the high-dose } \\
\text { treatment } \\
\text { (Continued on following page) }\end{array}$ \\
\hline
\end{tabular}


TABLE 2 | (Continued) Studies evaluating the impact of HBOCs in solid organ transplantation.

\begin{tabular}{|c|c|c|c|c|c|c|c|c|c|}
\hline $\begin{array}{l}\text { Author } \\
\text { and year }\end{array}$ & $\begin{array}{l}\text { Oxygen } \\
\text { carrier }\end{array}$ & $\begin{array}{l}\text { Evaluated } \\
\text { solution }\end{array}$ & $\begin{array}{l}\text { Concen- } \\
\text { tration }\end{array}$ & $\begin{array}{l}\text { Oxyge- } \\
\text { nation }\end{array}$ & $\begin{array}{l}\text { Experimental } \\
\text { proposal }\end{array}$ & Species & Results & $\begin{array}{l}\text { Author } \\
\text { conclusion }\end{array}$ & Limitation/toxicity \\
\hline & & & & & & & $\begin{array}{l}\text { improved islet graft } \\
\text { function, and } \\
\text { reduced the number } \\
\text { of islets needed for } \\
\text { successful } \\
\text { intramuscular } \\
\text { transplantation }\end{array}$ & & \\
\hline $\begin{array}{l}\text { Lemaire et al. (2019) } \\
\text { J Cell Mol Med } 2019\end{array}$ & M101 & $2 \mathrm{~g} / \mathrm{L}$ & $\begin{array}{l}\text { Hanks' } \\
\text { Balanced } \\
\text { Salt Solution } \\
\text { (HBSS) }\end{array}$ & $\mathrm{N}$ & $\begin{array}{l}\text { Cold storage (HBSS } \\
+ \text { M101 0, 2, 4, 6, } 8 \text {, } \\
\text { 12, and } 18 \text { h); } \\
\text { Perfusion: } 4^{\circ} \mathrm{C} 6 \mathrm{~h} \\
\text { with M101 or without } \\
\text { M101 }\end{array}$ & Wistar rat & $\begin{array}{l}\text { M101 decreased } \\
\text { oxidative stress } \\
\text { (ROS) and necrosis } \\
\text { (HMGB1); cellular } \\
\text { stress pathway (p38 } \\
\text { MAPK) activity was } \\
\text { observed; improved } \\
\text { post-isolation islet } \\
\text { quality }\end{array}$ & $\begin{array}{l}\text { Maintained oxidative } \\
\text { phosphorylation; } \\
\text { improved organ } \\
\text { oxygenation; intrinsic } \\
\text { SOD-like activity }\end{array}$ & $\mathrm{N}$ \\
\hline $\begin{array}{l}\text { Lemaire et al. (2019) } \\
\text { J Cell Mol Med } 2019\end{array}$ & M101 & $2 \mathrm{~g} / \mathrm{L}$ & UW & $\mathrm{N}$ & $\begin{array}{l}\text { Cold storage (SCS } \\
6 \mathrm{~h}+\mathrm{M} 1013 \mathrm{~h} \text { ) }\end{array}$ & Human & $\begin{array}{l}\text { M101 increased } \\
\text { complex } 1 \\
\text { mitochondrial } \\
\text { activity and } \\
\text { activated AKT } \\
\text { activity; upregulated } \\
\text { insulin secretion }\end{array}$ & $\begin{array}{l}\text { Maintained oxidative } \\
\text { phosphorylation; } \\
\text { improved organ } \\
\text { oxygenation; intrinsic } \\
\text { SOD-like activity }\end{array}$ & $\mathrm{N}$ \\
\hline \multicolumn{10}{|l|}{ Intestine } \\
\hline $\begin{array}{l}\text { Huang et al. (2014) } \\
\text { Artif Cells Nanomed } \\
\text { Biotechnol } 2014\end{array}$ & pPolyHb & UW & $\begin{array}{l}10.5 \pm \\
0.5 \mathrm{~g} / \mathrm{dl}\end{array}$ & $\begin{array}{l}\text { Equilibrated } \\
\text { with } 95 \% \mathrm{O}_{2} \\
\text { and } 5 \% \mathrm{CO}_{2} \\
\text { for } 30 \mathrm{~min}\end{array}$ & $\begin{array}{l}\text { Group 1: UW solution } \\
\text { (SCS 12, 24, and } \\
36 \text { h); Group 2: HCA } \\
\text { solution with } 2 \text { g/dl } \\
\text { pPolyHb (SCS 12, } \\
\text { 24, and } 36 \text { h); Group } \\
\text { 3: HCA solution with } \\
4 \text { g/dl pPolyHb (SCS } \\
\text { 12, 24, and } 36 \text { h); } \\
\text { Group 4: HCA } \\
\text { solution (SCS 12, 24, } \\
\text { and } 36 \text { h) }\end{array}$ & $\begin{array}{l}\text { Sprague-Dawley } \\
\text { rats }\end{array}$ & $\begin{array}{l}\text { Long-term (36 h) } \\
\text { morphological } \\
\text { integrity of the } \\
\text { intestinal mucosa } \\
\text { was better } \\
\text { preserved in the } \\
\text { pPolyHb; } \\
\text { maintained tissue } \\
\text { aerobic respiration } \\
\text { and inhibited tissue } \\
\text { anaerobic } \\
\text { metabolism }\end{array}$ & $\begin{array}{l}\text { Improved organ } \\
\text { oxygenation }\end{array}$ & $\mathrm{N}$ \\
\hline
\end{tabular}

STS, St. Thomas' solution; HS, hypothermic storage; CS, cold storage; NMP, normothermic machine perfusion; NO, nitric oxide; KHB, Krebs-Henseleit buffer; WI, warm ischemia; SOD, superoxide dismutase; HTK,

histidine-tryptophan-ketoglutarate; KH-Sol, Krebs-Henseleit solution; ROS, reactive oxygen species; TLR, toll-like receptor; RBC, red blood cell; PRBC, packed red blood cells; CVR, cerebrovascular resistance; $L P D$, low-potassium dextran solution; EVLP, ex vivo lung perfusion; SnPP, Sn-protoporphyrin; HO-1, heme oxygenase-1; IPRL, isolated perfused rat liver; UW, University of Wisconsin; MP, machine perfusion; FFP, fresh frozen plasma; DHOPE, hypothermic oxygenated machine perfusion; COR, controlled oxygenated rewarming; SCS, static cold storage; ATP, adenosine triphosphate; SNMP, subnormothermic machine perfusion; HMP, hypothermic mechanical perfusion; KPS, kidney preservation solution; SFH-P, stroma-free hemoglobin pyridoxalated; HBSS, Hanks' Balanced Salt Solution; HMGB1, high mobility group box; AKT, serine threonrine kinase; HCA, hypertonic citrate adenine. 
in mitochondria of isolated heart tissue, which delays the damage of ischemia and hypoxia and extends the cold storage time (Teh et al., 2017). Li et al. were the first to find that PolyPHb protected isolated hearts from IRI, which was related to a reduction in NOmediated myocardial apoptosis and restoration of the nitrosoredox balance ( $\mathrm{Li}$ et al., 2009). PolyPHb also improved mitochondrial function and maintained the mitochondrial structure, which indicated that it played an important role in maintaining redox homeostasis and reducing mitochondrial oxidative damage ( $\mathrm{Li}$ et al., 2010). The pathogenesis of IRI is complex, and inhibition of the inflammatory response is a reasonable way to ameliorate IRI. PolyPHb protected isolated hearts from cold IRI by attenuating the expression of toll-like receptor 2 (TLR2) and toll-like receptor 4 (TLR4)/nuclear factor kappa B (NF-kB) signaling pathways (Wei et al., 2011). The addition of M101 to Celsior preservation solution significantly improved cardiac function recovery after ischemia, and the coronary blood flow and left ventricular development pressure (LVDP) recovered significantly after $60 \mathrm{~min}$ of reperfusion (Teh et al., 2017).

The studies mentioned above confirmed that HBOCs reduced the IRI of the isolated heart and improved aerobic metabolism and myocardial function. However, an HBOC-201-based perfusate restored myocardial energy storage and extracted oxygen faster than blood-based perfusion during ex vivo heart perfusion (EVHP) to meet the needs of myocardial metabolism, but it did not minimize myocardial injury or provide superior preservation of myocardial function (White et al., 2015). These effects may be due to HBOC-201-mediated reactive oxygen species (ROS) generation and the increase in methemoglobin (White et al., 2015), which caused cardiac endothelial cell injury and increased microvascular permeability and coronary vascular resistance (CVR) and ultimately led to myocardial edema and cardiac diastolic dysfunction. HBOC-201 may also have a unique mechanism of myocardial injury that leads to increased levels of troponin I and histological myocardial injury scores (White et al., 2015). Whether reducing the dose of HBOC-201 and incorporating additional antioxidants will optimize the preservation of the donor's heart during EVHP is unclear and needs further verification in larger studies.

\section{Lung}

Lung transplantation has been the most effective treatment for most end-stage lung diseases since 1983 (Reitz et al., 1983). IRI is the main reason for primary graft dysfunction (PGD), which is the major cause of mortality and morbidity in the postoperative period. During SCS of the isolated lung, high oxygen content in the alveoli increases the lipid peroxidation rate, and low oxygen content may lead to tissue hypoxia (Fukuse et al., 2001). In addition, the ideal condition for preserving the isolated lung is to provide enough oxygen to maintain the aerobic metabolism of the cells while minimizing hyperoxia-related cellular oxidant injury. M101 does not require any allosteric effector to release oxygen molecules in an oxygen gradient passively, and it provides an appropriate amount of oxygen to the tissues. Glorion et al. first used M101 as an additive to the standard preservation solution to prevent IRI during $24 \mathrm{~h}$ static cold preservation in a well- recognized single-lung allotransplantation model in pigs (Glorion et al., 2018). The functional parameters in the bronchoalveolar lavage fluid (BALF) and the arterial partial pressure of oxygen to fraction of inspired oxygen ratio $\left(\mathrm{PaO}_{2} /\right.$ $\mathrm{FiO}_{2}$ ) of the graft in the M101 group were significantly improved, and the graft vascular resistance was reduced after $5 \mathrm{~h}$ of reperfusion in the recipient. The study identified a circulating biomarker of ischemia-reperfusion, high mobility group box 1 protein (HMGB1). The expression of HMGB1 in the serum of the M101 group tended to be lower, and the activation of the HMGB1/RAGE (advanced glycation end products) axis in the control group explained the inflammatory response that led to PDG. The early serious events that occurred in the first few hours after transplantation were sufficient to capture the development of PDG. Therefore, the protective effect of M101 on IRI prevented the occurrence of PGD. Another study reported that SCS for up to $36 \mathrm{~h}$ after adding M101 reduced the risk of PGD after lung transplantation, which may be related to a significant decrease in circulating interleukin-6 (IL-6) levels (Ali et al., 2020). Compared to the standard lung preservation program, the isolated lung was washed and preserved with an M101 preservation solution and evaluated using ex vivo lung perfusion (EVLP) for $12 \mathrm{~h}$; the M101 lung showed better lung function after transplantation. M101 has inherent oxidative stress reduction activity, but it produces no significant difference in oxidative injury. The effect of M101 in prolonging the isolated lung's preservation time may not be as an antioxidant, but it may optimize tissue oxygenation in the area of atelectasis. Briefly, adding M101 preservation solution prolongs the preservation time of isolated lungs up to $48 \mathrm{~h}$, which will help overcome longdistance obstacles and expand the donor pool.

\section{Liver}

Liver graft quality depends on the donation status of the donor and the preservation effect during transportation from the donor to the recipient. During the preservation of the isolated liver, insufficient oxygen supply followed by reperfusion leads to an inflammatory network, and oxidative stress in the tissue leads to IRI. HBOCs induce the production of heme oxygenase 1 (HO-1) (Damle et al., 2009). Topp and Donner et al. confirmed that the positive effect of reducing isolated liver IRI depended on the induction of HO-1, which may be inhibited by tin protoporphyrin IX dichloride (SnPP) (Donner et al., 2013; Topp, et al., 2008). By inducing HO-1, HbG200 largely restored multidrug resistance-associated protein 2 (Mrp2) and bile salt export pump (Bsep) in pericentral hepatocytes and improved bile flow and biliary taurocholate excretion, which are related to diminished hepatocellular c-Jun $\mathrm{N}$-terminal kinase (JNK) and Fyn signaling. Biliverdin/bilirubin and CO, which are the breakdown products of HO-1, also regulate JNK activity. Compared to traditional static cold preservation, both hypothermic oxygenated machine perfusion (HOPE) and SCS + M101 effectively oxygenated and increased ATP synthesis during the preservation of the isolated liver and prevented injury after liver transplantation (Alix et al., 2020). This study showed that HOPE had better results than M101 supplements, which may be related to the constant supply of oxygen and continuous capillary 
network flushing during liver perfusion. Nevertheless, SCS + M101 preservation for non-high-risk donor livers will be more convenient and easier to implement due to the high cost, manpower support, and risks of vascular injury associated with the use of HOPE. Compared to the current standard-of-care (CSP) system, the SNMP/HBOC system also significantly improved preservation of the isolated liver and provided superior graft function and $100 \%$ survival after transplantation, which may be further extended to the recovery and improvement of grafts obtained by ECDs (Fontes et al., 2015). In a pig model of DCD, HbV significantly increased the oxygen consumption of the donor's liver during SNMP, but it did not prevent liver injury because the amount of $\mathrm{HbV}$ (0.6-0.7 g/dl) was insufficient (Shonaka et al., 2018). However, another study showed that SNMP + HbV solution reduced reperfusion injury in DCD donor livers (Shonaka et al., 2019). The mitochondria, $\mathrm{pH}$, and lactate levels of the SNMP $+\mathrm{HbV}$ group were well maintained compared to the cold storage (CS), HMP, and SNMP groups.

NMP is generally performed using perfusion solutions based on RBCs to maintain physiological osmolarity, oncotic pressure, and adequate metabolic support. Laing et al. presented the first experience using an HBOC-based perfusion fluid to perfuse five discarded high-risk human livers in a human model of NMP (Laing et al., 2017). Livers perfused using the NMP/HBOC system had similar vascular flow parameters and perfused lactate clearance as the RBC-based perfusate but extracted more oxygen, which may be more beneficial in logistics, rheology, and immunology (Laing et al., 2017). Therefore, HBOC-201 may be used as an alternative oxygen carrier for RBCs in NMP. Matton et al. also reported that HBOC-201-based perfusate could be effective for NMP by using HBOC-201 + gelofusine perfusate instead of RBCs + FFP perfusate, while the results of some biomarkers of liver function and injury were even better without the use of human blood products (Matton et al., 2018). COR mitigates reperfusion injury and improves graft function posttransplantation (Hoyer et al., 2016). Boteon et al. reported that after perfusion of human high-risk donor livers using a combined cold-to-warm uninterrupted combined protocol (a single HBOC-based perfusate) and the HOPE + NMP interrupted combined protocol, both protocols reduced oxidation-mediated tissue injury and enhanced hepatic energy stores (Boteon et al., 2019). It showed that the combined protocol of $\mathrm{COR}$ and $\mathrm{HBOC}$ is beneficial for repairing high-risk donor livers while also eliminating the additional ischemic time required for perfusate exchange and simplifying the logistics of this combination, which may benefit its clinical application. de Vries et al. first reported that after serial DHOPE-COR-NMP using HBOC-201-based perfusate in seven high-risk donor livers, five of them were transplanted into recipients after evaluation that met all of the following transplantation criteria: within $150 \mathrm{~min}$ of NMP perfusate lactate $<1.7 \mathrm{mmol} / \mathrm{L}$, pH 7.35-7.45, bile production $>10 \mathrm{ml}$, and bile $\mathrm{pH}>7.45$ (de Vries et al., 2019). Similarly, in a prospective clinical trial by van et al., after perfusion of 16 high-risk donor livers with HBOC-201-based perfusion solution combined with DHOPE-COR-NMP, 11 of these donor's livers were transplanted into recipients after repair and evaluation of the liver and the survival of patients and grafts was $100 \%$ at 6 months (van Leeuwen et al., 2019). The study ultimately showed that the introduction of HBOCs in combination with DHOPE-COR-NMP could increase the number of high-risk donor liver transplants by $20 \%$ at the center. HBOC in combination with MP has been applied to the preservation of high-risk donor livers in clinical settings and has achieved a relatively favorable prognosis. However, randomized clinical trials with larger samples should be implemented to verify the benefits and prospects they offer, and the longer-term prognosis of the recipients should continue to be closely observed.

\section{Kidney}

Kidney transplantation is the therapy of choice for end-stage renal diseases. IRI is a complex process that involves oxidative stress, mitochondrial uncoupling, and the coagulation cascade, which affect early graft function after organ transplantation and have an adverse effect on the long-term survival of grafts (Favreau et al., 2010). Thuillier et al. found that supplementation of M101 in UW or HTK solution reduced apoptosis and maintained mitochondria in a time- and dose-dependent manner during cold preservation in a pig kidney auto-transplantation model, with significant benefits in both early functional recovery and prognosis after transplantation, and reduced the occurrence of IRI and interstitial fibrosis and tubular atrophy (IFTA) (Thuillier et al., 2011). Similarly, Mallet et al. also presented a dosedependent reduction in the extent of IRI and improved prognosis of grafts after cold preservation of porcine kidneys with UW solution supplemented with M101, and benefits were also obtained with lower doses of M101 ( $1 \mathrm{~g} / \mathrm{L})$ (Mallet et al., 2014). As high-risk donor kidneys are more prone to severe ischemia-reperfusion injury, there is an increased probability of delayed injury and dysfunction in the recipient and the graft (Oberbauer, 2016). As the demand for organ transplantation increases, repairing and assessing the quality of high-risk donor organs and improving the utilization of high-risk organs are among current solutions to expand the number of transplantable organs. Preservation of high-risk donor livers using SCS + M101 also showed dose-dependent improvement in long-term graft function; similarly, combining M101 with HMP had an improved effect on both short- and long-term graft function after transplantation (Kaminski et al., 2019). The combination of MP with HBOC allows for a uniform distribution of the HBOC in the vascular lumen and a more efficient delivery of oxygen to the tissues, further reducing ischemic and hypoxic injury and repairing the graft mass. HBOCs improve tissue oxygenation and repair kidney quality by releasing oxygen. However, if pure oxygen is added to the preservation solution for organ preservation, will the expected benefits be consistent? Kasil et al. evaluated the effect of supplemental oxygen [ $(100 \%$ $\left.\mathrm{O}_{2}\right) \pm \mathrm{M} 101$ (respectively or combined)] with HMP on the perfusion of isolated kidneys in a pig kidney autotransplantation model (Kasil et al., 2019). The results showed that using $100 \% \mathrm{O}_{2}$ in $\mathrm{HMP}$ without supplementation with $\mathrm{M} 101$ was harmful to the kidneys, which manifested as delayed vimentin expression and did not limit renal fibrosis. In 
addition, the combination of $\mathrm{M} 101$ and $100 \% \mathrm{O}_{2}$ reduced the level of neutrophil gelatinase-associated lipocalin (NGAL) and maintained a high level of kidney injury molecule-1 (KIM-1) in the circulation. The study showed that supplementation with M101 with or without $100 \% \mathrm{O}_{2}$ improves the HMP effect on kidney recovery and late graft results.

Gradual rewarming is a new perfusion modality in which HBOC maintains the preservation of perfusion of organs from hypothermia to normothermia. A transient increase in vascular resistance and methemoglobin was observed after preservation of the kidney using a preservation solution supplemented with HBOC-201 in a model of gradual rewarming but improved ultrafiltrate production, glomerular filtration rate, and sodium reabsorption during the reperfusion phase (Mahboub et al., 2020). In the context of combination with MP, the risk of HBOC-201 causing a transient increase in vascular resistance and methemoglobin transfer to the organ recipient is negligible because HBOC and methemoglobin will be washed out before transplantation. Perfusion preservation of organs using blood cannot be performed at low temperatures because it can lead to hemolysis and impair the quality of the grafts. Bhattacharjee et al. perfused porcine DCD kidneys with HBOC-201 or blood in combination with SNMP and found that tissue oxygen saturation, renal blood flow, and function were similar in both groups (Bhattacharjee et al., 2020). Thus, this demonstrated that HBOC-201 is a suitable oxygen carrier capable of replacing blood. NMP is capable of perfusing and repairing organs in combination with oxygen carriers and assessing the quality of the grafts. Mohamed et al. used HBOCs or packed red blood cells (PRBC) combined with NMP for perfusion and evaluation of human high-risk donor kidneys and found that the function and histological characteristics of the kidney were similar (Aburawi et al., 2019). This study confirmed that the HBOC solution could provide a logistically convenient alternative to PRBC in the NMP of human kidneys. Meur et al. demonstrated for the first time the safety and performance of the addition of M101 to the preservation solution of one of the two human kidneys from the same donor in a multicenter open-label study (Le Meur et al., 2020). This study showed that kidneys preserved with the addition of M101 showed no alterations in thrombosis or microcirculation on biopsy before implantation and 3 months after transplantation and had significantly less DGF and better kidney function after transplantation. The use of M101 for kidney preservation has been applied clinically and has confirmed the safety and promising efficacy data of M101 (Le Meur et al., 2020).

\section{Pancreas and Small Bowel}

Pancreas or islet transplantation is a treatment for type 1 diabetes and pancreatitis (Barrou et al., 1994). During the preservation and isolation of islets, maintenance of proper oxygen levels is of great importance to prevent ischemia and reperfusion injury (Carlsson and Palm, 2002). Avila et al. showed that intratubular perfusion of HBOCs in the pancreas of rats undergoing $30 \mathrm{~min}$ of warm ischemia improved islet isolation and transplantation outcomes via maintaining mitochondrial integrity and did not lead to increased oxidative stress (Avila et al., 2006). M101 was added to the preservation solution during cold preservation of rat and human pancreas, and hypoxia and the oxidative stress of SOD were reduced, which alleviated tissue inflammation and necrosis (Lemaire et al., 2019). Overall, these results suggested that M101 had a positive effect on preserving the pancreas in rats and humans.

Muscle is a promising alternative site for islet transplantation, facilitating the rapid restoration of islet vasculature; however, it is susceptible to hypoxic limitation in the early posttransplant period. By co-transplanting islets with an oxygen carrierPolyHb in mice, Daniel et al. found a dose-dependent reduction in $\beta$-cell hypoxia (Espes et al., 2015). Yet, the morphology of islets was significantly disrupted by the higher concentration of a bovine polyhemoglobin (PolyHb) inducing the aggregation of a large number of macrophages, and the reduction in $\beta$-cell apoptosis occurred only in the low-dose group $(0.03 \mathrm{mg}$ / g body weight). Lower doses of PolyHb co-transplanted with islets effectively bridged the critical hypoxic phase after transplantation, improved islet graft function, and reduced the number of islets required for successful intramuscular transplantation. In contrast, the inflammatory effect of higher concentrations of PolyHb may be due to the attraction of macrophages to the $\mathrm{Hb}$-haptoglobin complex. HBOCs have also been used in rat small bowel preservation (Huang et al., 2014). This study suggested that hypertonic adenine citrate solution (HCA) combined with pPolyHb maintained tissue aerobic respiration and inhibited anaerobic metabolism with a preservation effect comparable to that of UW solution.

\section{OVERALL CONCLUSION AND FUTURE PERSPECTIVES}

Due to the global shortage of organ resources, the imbalance of donor-recipient needs, and the increasing number of high-risk donors, these issues have led to considering what should be done to preserve grafts better. Although standard preservation solutions are currently used for static cold preservation, the repair and evaluation of marginal organs may rely on multiple combinations of MP, oxygenation strategies, and temperature regulation. Many efforts have been made by clinical practitioners and researchers to explore ways to mitigate graft ischemia-reperfusion injury, improve graft quality, and increase recipient and graft survival rates. Originally developed and utilized as a blood substitute, HBOCs are an ideal class of oxygen carriers with the ability to bind oxygen and release oxygen. HBOCs have now been extended to apply to tissues or organs in hypoxic conditions, where they act as an oxygen bridge to improve tissue oxygenation. The addition of HBOC to the preservation solution to improve the quality of the graft is a new promising strategy because they offer several advantages compared with traditional preservation solutions. First, HBOCs improve the oxygenation of isolated organs during SCS and enhance the quality of grafts. Second, the combination of HBOCs and MP provides a more targeted method, which can replace $\mathrm{RBC}$ perfusion to improve and evaluate the quality of high-risk grafts. In addition, preservation solutions containing HBOCs may be easily used in all solid organ transplants without 
further improving the existing preservation solution or perfusion platform protocols. Recent clinical trials have demonstrated the safety and efficacy of HBOCs in combination with mechanical perfusion platforms for liver and kidney preservation and improved prognosis for recipients and grafts.

Future prospects address the need for translational medicine research to determine the exact therapeutic dose and organ preservation time for human solid organs. Coordinated multicenter clinical trials are also needed in the subsequent clinical studies to accumulate larger, homogeneous cohorts. Additionally, some clinical trials with a randomized controlled design would be performed. This review summarizes the latest and most important research on HBOCs in solid organ preservation. Therefore, based on scientific evidence, HBOCs, one of the oxygen carriers for organ preservation, are ideal candidates for organ preservation additives. The mechanism of organ preservation by HBOCs has not been fully understood; the relevant mechanism would be investigated in depth in future studies to determine a more optimal preservation method. In addition, the vascular resistance, methemoglobin content, and oxidative damage index of grafts and recipients would also be closely monitored when using HBOCs. The future development

\section{REFERENCES}

Aburawi, M. M., Fontan, F. M., Karimian, N., Eymard, C., Cronin, S., Pendexter, C., et al. (2019). Synthetic Hemoglobin-Based Oxygen Carriers Are an Acceptable Alternative for Packed Red Blood Cells in Normothermic Kidney Perfusion. Am. J. Transpl. 19, 2814-2824. doi:10.1111/ajt.15375

Adachi, K., Kim, J., Travitz, R., Harano, T., and Asakura, T. (1987). Effect of Amino Acid at the Beta 6 Position on Surface Hydrophobicity, Stability, Solubility, and the Kinetics of Polymerization of Hemoglobin. Comparisons Among $\mathrm{Hb} \mathrm{A}$ (Glu Beta 6), Hb C (Lys Beta 6), Hb Machida (Gln Beta 6), and Hb S (Val Beta 6). J. Biol. Chem. 262, 12920-12925. doi:10.1016/s0021-9258(18)45145-9

Alayash, A. I., D’Agnillo, F., and Buehler, P. W. (2007). First-generation Blood Substitutes: what Have We Learned? Biochemical and Physiological Perspectives. Expert Opin. Biol. Ther. 7, 665-675. doi:10.1517/14712598.7.5.665

Alayash, A. I. (2017). Hemoglobin-Based Blood Substitutes and the Treatment of Sickle Cell Disease: More Harm Than Help. Biomolecules 7, 2. doi:10.3390/ biom7010002

Ali, A., Watanabe, Y., Galasso, M., Watanabe, T., Chen, M., Fan, E., et al. (2020). An Extracellular Oxygen Carrier during Prolonged Pulmonary Preservation Improves post-transplant Lung Function. J. Heart Lung Transpl. 39, 595-603. doi:10.1016/j.healun.2020.03.027

Alix, P., Val-Laillet, D., Turlin, B., Ben Mosbah, I., Burel, A., Bobillier, E., et al. (2020). Adding the Oxygen Carrier M101 to a Cold-Storage Solution Could Be an Alternative to HOPE for Liver Graft Preservation. JHEP Rep. 2, 100119. doi:10.1016/j.jhepr.2020.100119

Avila, J. G., Wang, Y., Barbaro, B., Gangemi, A., Qi, M., Kuechle, J., et al. (2006). Improved Outcomes in Islet Isolation and Transplantation by the Use of a Novel Hemoglobin-Based O2 Carrier. Am. J. Transpl. 6, 2861-2870. doi:10.1111/j.1600-6143.2006.01551.x

Barrou, Z., Seaquist, E. R., and Robertson, R. P. (1994). Pancreas Transplantation in Diabetic Humans Normalizes Hepatic Glucose Production during Hypoglycemia. Diabetes 43, 661-666. doi:10.2337/diab.43.5.661

Belcher, D. A., Lucas, A., Cabrales, P., and Palmer, A. F. (2020). Tumor Vascular Status Controls Oxygen Delivery Facilitated by Infused Polymerized Hemoglobins with Varying Oxygen Affinity. Plos Comput. Biol. 16, e1008157. doi:10.1371/journal.pcbi.1008157

Bhattacharjee, R. N., Patel, S. V. B., Sun, Q., Jiang, L., Richard-Mohamed, M., Ruthirakanthan, A., et al. (2020). Renal Protection against Ischemia Reperfusion Injury: Hemoglobin-Based Oxygen Carrier-201 versus Blood as of HBOCs may give a fascinating insight into the understanding of the safety and effectiveness of new strategies for solid organ preservation.

\section{AUTHOR CONTRIBUTIONS}

$\mathrm{MC}, \mathrm{GW}$, and $\mathrm{HH}$ were responsible for the organization and preliminary analysis of the literature; RY, YZ, LP, WH, YG, TY, and LM were involved in the data collection and collation; MC, $\mathrm{DZ}$, and $\mathrm{XH}$ were responsible for the conceptualization and writing of the original draft; $\mathrm{DZ}$ and $\mathrm{XH}$ contributed to the final edits and general revisions. All authors have read and approved the final version of the manuscript.

\section{FUNDING}

This work was supported by the National Natural Science Foundation of China (81470667), Department of Science and Technology of Sichuan Province (20YFS0435), and Science \& Technology Bureau of Chengdu (YF06-00070-SN and YF05-00198-SN).

an Oxygen Carrier in Ex Vivo Subnormothermic Machine Perfusion. Transplantation 104, 482-489. doi:10.1097/TP.0000000000002967

Bleeker, W. K., van der Plas, J., Feitsma, R. I., Agterberg, J., Rigter, G., de Vries-van Rossen, A., et al. (1989). In Vivo distribution and Elimination of Hemoglobin Modified by Intramolecular Cross-Linking with 2-Nor-2-Formylpyridoxal 5'phosphate. J. Lab. Clin. Med. 113, 151-161.

Bobofchak, K. M., Mito, T., Texel, S. J., Bellelli, A., Nemoto, M., Traystman, R. J., et al. (2003). A Recombinant Polymeric Hemoglobin with Conformational, Functional, and Physiological Characteristics of an In Vivo O2 Transporter. Am. J. Physiol. Heart Circ. Physiol. 285, H549-H561. doi:10.1152/ ajpheart.00037.2003

Boteon, Y. L., Laing, R. W., Schlegel, A., Wallace, L., Smith, A., Attard, J., et al. (2019). The Impact on the Bioenergetic Status and Oxidative-Mediated Tissue Injury of a Combined Protocol of Hypothermic and Normothermic Machine Perfusion Using an Acellular Haemoglobin-Based Oxygen Carrier: The ColdTo-Warm Machine Perfusion of the Liver. PLoS One 14, e224066. doi:10.1371/ journal.pone.0224066

Cabrales, P., Sun, G., Zhou, Y., Harris, D. R., Tsai, A. G., Intaglietta, M., et al. (2009). Effects of the Molecular Mass of Tense-State Polymerized Bovine Hemoglobin on Blood Pressure and Vasoconstriction. J. Appl. Physiol. 107, 1548-1558. doi:10.1152/japplphysiol.00622.2009

Cai, Y., Dai, Z., Wen, S., and Bhandari, R. (2020). Risk Factors Associated with Infection of Blood-Borne Virus Among People Who Used Methamphetamine. BMC Infect. Dis. 20, 742. doi:10.1186/s12879-020-05464-y

Callas, D. D., Clark, T. L., Moreira, P. L., Lansden, C., Gawryl, M. S., Kahn, S., et al. (1997). In Vitro effects of a Novel Hemoglobin-Based Oxygen Carrier on Routine Chemistry, Therapeutic Drug, Coagulation, Hematology, and Blood Bank Assays. Clin. Chem. 43, 1744-1748. doi:10.1093/clinchem/ 43.9.1744

Carlsson, P. O., and Palm, F. (2002). Oxygen Tension in Isolated Transplanted Rat Islets and in Islets of Rat Whole-Pancreas Transplants. Transpl. Int. 15, 581-585. doi:10.1007/s00147-002-0460-7

Caswell, J. E., Strange, M. B., Rimmer, D. M., 3rd, Gibson, M. F., Cole, P., and Lefer, D. J. (2005). A Novel Hemoglobin-Based Blood Substitute Protects against Myocardial Reperfusion Injury. Am. J. Physiol. Heart Circ. Physiol. 288, H1796-H1801. doi:10.1152/ajpheart.00905.2004

Damle, S. S., Moore, E. E., Babu, A. N., Meng, X., Fullerton, D. A., and Banerjee, A. (2009). Hemoglobin-based Oxygen Carrier Induces Heme Oxygenase-1 in the Heart and Lung but Not Brain. J. Am. Coll. Surg. 208, 592-598. doi:10.1016/ j.jamcollsurg.2009.01.015 
de Vries, Y., Matton, A. P. M., Nijsten, M. W. N., Werner, M. J. M., van den Berg, A. P., de Boer, M. T., et al. (2019). Pretransplant Sequential Hypo- and Normothermic Machine Perfusion of Suboptimal Livers Donated after Circulatory Death Using a Hemoglobin-Based Oxygen Carrier Perfusion Solution. Am. J. Transpl. 19, 1202-1211. doi:10.1111/ajt.15228

Dixit, V. A., Blumberger, J., and Vyas, S. K. (2021). Methemoglobin Formation in Mutant Hemoglobin a Chains: Electron Transfer Parameters and Rates. Biophys. J. 120, 3807-3819. doi:10.1016/j.bpj.2021.07.007

Donner, M. G., Topp, S. A., Cebula, P., Krienen, A., Gehrmann, T., Sommerfeld, A., et al. (2013). HbG200-mediated Preinduction of Heme Oxygenase-1 Improves Bile Flow and Ameliorates Pericentral Downregulation of Bsep and Mrp2 Following Experimental Liver Ischemia and Reperfusion. Biol. Chem. 394, 97-112. doi:10.1515/hsz-2012-0153

Doyle, M. P., and Hoekstra, J. W. (1981). Oxidation of Nitrogen Oxides by Bound Dioxygen in Hemoproteins. J. Inorg. Biochem. 14, 351-358. doi:10.1016/s01620134(00)80291-3

Dubé, G. P., Vranckx, P., and Greenburg, A. G. (2008). HBOC-201: the Multi-Purpose Oxygen Therapeutic. EuroIntervention 4, 161-165. doi:10.4244/eijv4ila26

Espes, D., Lau, J., Quach, M., Banerjee, U., Palmer, A. F., and Carlsson, P. O. (2015). Cotransplantation of Polymerized Hemoglobin Reduces $\beta$-Cell Hypoxia and Improves $\beta$-Cell Function in Intramuscular Islet Grafts. Transplantation 99, 2077-2082. doi:10.1097/TP.0000000000000815

Favreau, F., Thuillier, R., Cau, J., Milin, S., Manguy, E., Mauco, G., et al. (2010). Anti-thrombin Therapy during Warm Ischemia and Cold Preservation Prevents Chronic Kidney Graft Fibrosis in a DCD Model. Am. J. Transpl. 10, 30-39. doi:10.1111/j.1600-6143.2009.02924.x

Fontes, P., Lopez, R., van der Plaats, A., Vodovotz, Y., Minervini, M., Scott, V., et al. (2015). Liver Preservation with Machine Perfusion and a Newly Developed Cell-free Oxygen Carrier Solution under Subnormothermic Conditions. Am. J. Transpl. 15, 381-394. doi:10.1111/ajt.12991

Fukuse, T., Hirata, T., Ishikawa, S., Shoji, T., Yoshimura, T., Chen, Q., et al. (2001). Optimal Alveolar Oxygen Concentration for Cold Storage of the Lung. Transplantation 72, 300-304. doi:10.1097/00007890-200107270-00024

Gao, S., Guan, Q., Chafeeva, I., Brooks, D. E., Nguan, C. Y., Kizhakkedathu, J. N., et al. (2015). Hyperbranched Polyglycerol as a Colloid in Cold Organ Preservation Solutions. PloS one 10, e0116595. doi:10.1371/journal.pone.0116595

Glorion, M., Polard, V., Favereau, F., Hauet, T., Zal, F., Fadel, E., et al. (2018). Prevention of Ischemia-Reperfusion Lung Injury during Static Cold Preservation by Supplementation of Standard Preservation Solution with HEMO2life ${ }^{\circledR}$ in Pig Lung Transplantation Model. Artif. Cell Nanomed Biotechnol 46, 1773-1780. doi:10.1080/21691401.2017.1392315

Gordon, J. E., Dare, M. R., and Palmer, A. F. (2005). Engineering Select Physical Properties of Cross-Linked Red Blood Cells and a Simple A Priori Estimation of Their Efficacy as an Oxygen Delivery Vehicle within the Context of a Hepatic Hollow Fiber Bioreactor. Biotechnol. Prog. 21, 1700-1707. doi:10.1021/ bp050204p

Gould, S. A., Moore, E. E., Hoyt, D. B., Burch, J. M., Haenel, J. B., Garcia, J., et al. (1998). The First Randomized Trial of Human Polymerized Hemoglobin as a Blood Substitute in Acute Trauma and Emergent Surgery. J. Am. Coll. Surg. 187, 113. doi:10.1016/s1072-7515(98)00095-7

Grundmann, R., Strümper, R., Eichmann, J., and Pichlmaier, H. (1977). The Immediate Function of the Kidney after 24-to 72-hr Preservation. Hypothermic Storage versus Mechanical Perfusion. Transplantation 23, 437-443. doi:10.1097/00007890-197705000-00009

Han, J. G., Yang, Q., Yao, X. Q., Kwan, Y. W., Shen, B., and He, G. W. (2009). Role of Large-Conductance Calcium-Activated Potassium Channels of Coronary Arteries in Heart Preservation. J. Heart Lung Transpl. 28, 1094-1101. doi:10.1016/j.healun.2009.06.011

Henderson, C. L., Anderson, C. M., Sorrells, D. L., Wilson, B. J., Dick, E. J., and DiGeronimo, R. J. (2004). The Use of a Hemoglobin-Based Oxygen-Carrying Solution (HBOC-201) for Extracorporeal Membrane Oxygenation in a Porcine Model with Acute Respiratory Distress Syndrome. Pediatr. Crit. Care Med. 5, 384-390. doi:10.1097/01.pcc.0000123544.46047.ba

Higuchi, T., Oto, T., Millar, I. L., Levvey, B. J., Williams, T. J., and Snell, G. I. (2006). Preliminary Report of the Safety and Efficacy of Hyperbaric Oxygen Therapy for Specific Complications of Lung Transplantation. J. Heart Lung Transpl. 25, 1302-1309. doi:10.1016/j.healun.2006.08.006
Hoyer, D. P., Mathé, Z., Gallinat, A., Canbay, A. C., Treckmann, J. W., Rauen, U., et al. (2016). Controlled Oxygenated Rewarming of Cold Stored Livers Prior to Transplantation: First Clinical Application of a New Concept. Transplantation 100, 147-152. doi:10.1097/TP.0000000000000915

Huang, H., Ma, J., Zhu, W., Sun, J., Yan, K., Song, B., et al. (2014). The Application of Polymerized Porcine Hemoglobin (pPolyHb) in the Rat Small Bowel Preservation. Artif. Cell Nanomed Biotechnol 42, 289-295. doi:10.3109/ 21691401.2013.845571

Hughes, G. S., Yancey, E. P., Albrecht, R., Locker, P. K., Francom, S. F., Orringer, E. P., et al. (1995). Hemoglobin-based Oxygen Carrier Preserves Submaximal Exercise Capacity in Humans. Clin. Pharmacol. Ther. 58, 434-443. doi:10.1016/ 0009-9236(95)90057-8

Ingemansson, R., Massa, G., Pandita, R. K., Sjöberg, T., and Steen, S. (1995). Perfadex Is superior to Euro-Collins Solution Regarding 24-hour Preservation of Vascular Function. Ann. Thorac. Surg. 60, 1210-1214. doi:10.1016/00034975(95)00548-Y

Ingemansson, R., Sjöberg, T., and Steen, S. (1996). Importance of Calcium in LongTerm Preservation of the Vasculature. Ann. Thorac. Surg. 61, 1158-1162. doi:10.1016/0003-4975(95)01141-2

Israni, A. K., Zaun, D., Rosendale, J. D., Schaffhausen, C., McKinney, W., and Snyder, J. J. (2021). OPTN/SRTR 2019 Annual Data Report: Deceased Organ Donors. Am. J. Transpl. 21 (Suppl. 2), 567-604. doi:10.1111/ajt.16491

Jahr, J. S., Moallempour, M., and Lim, J. C. (2008). HBOC-201, Hemoglobin Glutamer-250 (Bovine), Hemopure (Biopure Corporation). Expert Opin. Biol. Ther. 8, 1425-1433. doi:10.1517/14712598.8.9.1425

Jeffers, A., Gladwin, M. T., and Kim-Shapiro, D. B. (2006). Computation of Plasma Hemoglobin Nitric Oxide Scavenging in Hemolytic Anemias. Free Radic. Biol. Med. 41, 1557-1565. doi:10.1016/j.freeradbiomed.2006.08.017

Kalisvaart, M., de Haan, J. E., Polak, W. G., N M IJzermans, J. J., Gommers, D., Metselaar, H. J., et al. (2018). Onset of Donor Warm Ischemia Time in Donation after Circulatory Death Liver Transplantation: Hypotension or Hypoxia. Liver Transplant. 24, 1001-1010. doi:10.1002/lt.25287

Kaminski, J., Hannaert, P., Kasil, A., Thuillier, R., Leize, E., Delpy, E., et al. (2019). Efficacy of the Natural Oxygen Transporter HEMO2 Life $^{\circledR}$ in Cold Preservation in a Preclinical Porcine Model of Donation after Cardiac Death. Transpl. Int. 32, 985-996. doi:10.1111/tri.13434

Kasil, A., Giraud, S., Couturier, P., Amiri, A., Danion, J., Donatini, G., et al. (2019). Individual and Combined Impact of Oxygen and Oxygen Transporter Supplementation during Kidney Machine Preservation in a Porcine Preclinical Kidney Transplantation Model. Int. J. Mol. Sci. 20, 1992. doi:10.3390/ijms20081992

Kim, J. D., Baker, C. J., Roberts, R. F., Darbinian, S. H., Marcus, K. A., Quardt, S. M., et al. (2000). Platelet Activating Factor Acetylhydrolase Decreases Lung Reperfusion Injury. Ann. Thorac. Surg. 70, 423-428. doi:10.1016/s00034975(00)01405-3

Kobayashi, T., Sumimoto, R., Shimada, H., Kamada, N., and Nakagawara, G. (1991). Effect of Sugars in the Preservation Solution on Liver Storage in Rats. Cryobiology 28, 428-435. doi:10.1016/0011-2240(91)90051-o

Laing, R. W., Bhogal, R. H., Wallace, L., Boteon, Y., Neil, D. A. H., Smith, A., et al. (2017). The Use of an Acellular Oxygen Carrier in a Human Liver Model of Normothermic Machine Perfusion. Transplantation 101, 2746-2756. doi:10.1097/TP.0000000000001821

Lavallard, V., Cottet-Dumoulin, D., Wassmer, C. H., Rouget, C., Parnaud, G., Brioudes, E., et al. (2020). NLRP3 Inflammasome Is Activated in Rat Pancreatic Islets by Transplantation and Hypoxia. Sci. Rep. 10, 7011. doi:10.1038/s41598020-64054-9

Le Meur, Y., Badet, L., Essig, M., Thierry, A., Büchler, M., Drouin, S., et al. (2020). First-in-human Use of a marine Oxygen Carrier (M101) for Organ Preservation: A Safety and Proof-Of-Principle Study. Am. J. Transpl. 20, 1729-1738. doi:10.1111/ajt.15798

Lemaire, F., Sigrist, S., Delpy, E., Cherfan, J., Peronet, C., Zal, F., et al. (2019). Beneficial Effects of the Novel marine Oxygen Carrier M101 during Cold Preservation of Rat and Human Pancreas. J. Cel Mol Med 23, 8025-8034. doi: $10.1111 /$ jcmm. 14666

Levien, L. J., Hodgson, R. E., and James, M. F. (2008). Hemoglobin-based Blood Substitutes and Risk of Myocardial Infarction and Death. JAMA 300, 1295-1299. doi:10.1001/jama.300.11.1295-a 
Li, T., Li, J., Liu, J., Zhang, P., Wu, W., Zhou, R., et al. (2009). Polymerized Placenta Hemoglobin Attenuates Ischemia/reperfusion Injury and Restores the NitrosoRedox Balance in Isolated Rat Heart. Free Radic. Biol. Med. 46, 397-405. doi:10.1016/j.freeradbiomed.2008.10.042

Li, T., Zhang, P., Liu, J., Zhou, R., Li, Q., You, Z., et al. (2010). Protective Effects of Hemoglobin-Based Oxygen Carrier Given to Isolated Heart during Ischemia via Attenuation of Mitochondrial Oxidative Damage. Free Radic. Biol. Med. 48 (48), 1079-1089. doi:10.1016/j.freeradbiomed.2010.01.027

Liu, L. P., Zhao, Q. Y., Wu, J., Luo, Y. W., Dong, H., Chen, Z. W., et al. (2021). Machine Learning for the Prediction of Red Blood Cell Transfusion in Patients during or after Liver Transplantation Surgery. Front. Med. (Lausanne) 8, 632210. doi:10.3389/fmed.2021.632210

Loscalzo, J. (1997). Nitric Oxide Binding and the Adverse Effects of Cell-free Hemoglobins: what Makes Us Different from Earthworms. J. Lab. Clin. Med. 129, 580-583. doi:10.1016/s0022-2143(97)90191-8

Lv, Y., Xiang, Q., Lin, J., Jin, Y. Z., Fang, Y., Cai, H. M., et al. (2021). There Is No Dose-Response Relationship between Allogeneic Blood Transfusion and Healthcare-Associated Infection: a Retrospective Cohort Study. Antimicrob. Resist. Infect. Control. 10, 62. doi:10.1186/s13756-021-00928-5

Mackenzie, C. F., Moon-Massat, P. F., Shander, A., Javidroozi, M., and Greenburg, A. G. (2010). When Blood Is Not an Option: Factors Affecting Survival after the Use of a Hemoglobin-Based Oxygen Carrier in 54 Patients with LifeThreatening Anemia. Anesth. Analg 110, 685-693. doi:10.1213/ ANE.0b013e3181cd473b

Mahboub, P., Aburawi, M., Karimian, N., Lin, F., Karabacak, M., Fontan, F., et al. (2020). The Efficacy of HBOC-201 in Ex Situ Gradual Rewarming Kidney Perfusion in a Rat Model. Artif. Organs 44, 81-90. doi:10.1111/aor.13534

Mallet, V., Dutheil, D., Polard, V., Rousselot, M., Leize, E., Hauet, T., et al. (2014). Dose-ranging Study of the Performance of the Natural Oxygen Transporter HEMO2 Life in Organ Preservation. Artif. Organs 38, 691-701. doi:10.1111/ aor.12307

Matton, A. P. M., Burlage, L. C., van Rijn, R., de Vries, Y., Karangwa, S. A., Nijsten, M. W., et al. (2018). Normothermic Machine Perfusion of Donor Livers without the Need for Human Blood Products. Liver Transpl. 24, 528-538. doi:10.1002/ lt. 25005

Mer, M., Hodgson, E., Wallis, L., Jacobson, B., Levien, L., Snyman, J., et al. (2016). Hemoglobin Glutamer-250 (Bovine) in South Africa: Consensus Usage Guidelines from Clinician Experts Who Have Treated Patients. Transfusion 56, 2631-2636. doi:10.1111/trf.13726

Minor, T., Koetting, M., Koetting, M., Kaiser, G., Efferz, P., Lüer, B., et al. (2011). Hypothermic Reconditioning by Gaseous Oxygen Improves Survival after Liver Transplantation in the Pig. Am. J. Transpl. 11, 2627-2634. doi:10.1111/j.16006143.2011.03731.x

Moore, K. P., Holt, S. G., Patel, R. P., Svistunenko, D. A., Zackert, W., Goodier, D., et al. (1998). A Causative Role for Redox Cycling of Myoglobin and its Inhibition by Alkalinization in the Pathogenesis and Treatment of Rhabdomyolysis-Induced Renal Failure. J. Biol. Chem. 273, 31731-31737. doi:10.1074/jbc.273.48.31731

Nakamura, K., Kageyama, S., Kaldas, F. M., Hirao, H., Ito, T., Kadono, K., et al. (2020). Hepatic CEACAM1 Expression Indicates Donor Liver Quality and Prevents Early Transplantation Injury. J. Clin. Invest. 130, 2689-2704. doi:10.1172/JCI133142

Natanson, C., Kern, S. J., Lurie, P., Banks, S. M., and Wolfe, S. M. (2008). Cell-free Hemoglobin-Based Blood Substitutes and Risk of Myocardial Infarction and Death: a Meta-Analysis. JAMA 299, 2304-2312. doi:10.1001/ jama.299.19.jrv80007

Oberbauer, R. (2016). Progression of Interstitial Fibrosis in Kidney Transplantation. Clin. J. Am. Soc. Nephrol. 11, 2110-2112. doi:10.2215/ CJN.09770916

OPTN/SRTR 2019 Annual Data Report (2021a). OPTN/SRTR 2019 Annual Data Report: Introduction. Am J Transplant. 21, 11-20. American Journal of Transplantation: Official Journal of the American Society of Transplantation and the American Society of Transplant Surgeons. doi:10.1111/ajt.16493

OPTN/SRTR 2019 Annual Data Report (2021b). OPTN/SRTR 2019 Annual Data Report: Preface. Am J Transplant. 21, 1-10. American Journal of Transplantation : Official Journal of the American Society of Transplantation and the American Society of Transplant Surgeons. doi: doi:10.1111/ajt.16497
Page, T. C., Light, W. R., McKay, C. B., and Hellums, J. D. (1998). Oxygen Transport by Erythrocyte/hemoglobin Solution Mixtures in an In Vitro Capillary as a Model of Hemoglobin-Based Oxygen Carrier Performance. Microvasc. Res. 55, 54-64. doi:10.1006/mvre.1997.2055

Partlow, K. C., Lanza, G. M., and Wickline, S. A. (2008). Exploiting Lipid Raft Transport with Membrane Targeted Nanoparticles: a Strategy for Cytosolic Drug Delivery. Biomaterials 29, 3367-3375. doi:10.1016/ j.biomaterials.2008.04.030

Pearce, L. B., and Gawryl, M. S. (2003). The Pharmacology of Tissue Oxygenation by Biopure's Hemoglobin-Based Oxygen Carrier, Hemopure (HBOC-201). Adv. Exp. Med. Biol. 530, 261-270. doi:10.1007/978-1-4615-0075-9_25

Reitz, B. A., Gaudiani, V. A., Hunt, S. A., Wallwork, J., Billingham, M. E., Oyer, P. E., et al. (1983). Diagnosis and Treatment of Allograft Rejection in Heart-Lung Transplant Recipients. J. Thorac. Cardiovasc. Surg. 85, 354-361. doi:10.1016/ s0022-5223(19)37565-8

Rother, R. P., Bell, L., Hillmen, P., and Gladwin, M. T. (2005). The Clinical Sequelae of Intravascular Hemolysis and Extracellular Plasma Hemoglobin: a Novel Mechanism of Human Disease. JAMA 293, 1653-1662. doi:10.1001/ jama.293.13.1653

Rousselot, M., Delpy, E., Drieu La Rochelle, C., Lagente, V., Pirow, R., Rees, J. F., et al. (2006). Arenicola marina Extracellular Hemoglobin: a New Promising Blood Substitute. Biotechnol. J. 1, 333-345. doi:10.1002/biot.200500049

Sahu, S. C., Simplaceanu, V., Gong, Q., Ho, N. T., Glushka, J. G., Prestegard, J. H., et al. (2006). Orientation of Deoxyhemoglobin at High Magnetic fields: Structural Insights from RDCs in Solution. J. Am. Chem. Soc. 128, 6290-6291. doi:10.1021/ja060023z

Sakai, H., Hamada, K., Takeoka, S., Nishide, H., and Tsuchida, E. (1996). Physical Properties of Hemoglobin Vesicles as Red Cell Substitutes. Biotechnol. Prog. 12, 119-125. doi:10.1021/bp950068w

Sakai, H., Horinouchi, H., Tomiyama, K., Ikeda, E., Takeoka, S., Kobayashi, K., et al. (2001). Hemoglobin-vesicles as Oxygen Carriers: Influence on Phagocytic Activity and Histopathological Changes in Reticuloendothelial System. Am. J. Pathol. 159, 1079-1088. doi:10.1016/S0002-9440(10)61783-X

Sakai, H., Tomiyama, K. I., Sou, K., Takeoka, S., and Tsuchida, E. (2000). Poly(ethylene Glycol)-Conjugation and Deoxygenation Enable Long-Term Preservation of Hemoglobin-Vesicles as Oxygen Carriers in a Liquid State. Bioconjug. Chem. 11, 425-432. doi:10.1021/bc990173h

Sakai, T., Li, S., Kuroda, Y., Tanioka, Y., Fujino, Y., and Suzuki, Y. (2011). Oxygenation of the portal Vein by Intraperitoneal Administration of Oxygenated Perfluorochemical Improves the Engraftment and Function of Intraportally Transplanted Islets. Pancreas 40, 403-409. doi:10.1097/ MPA.0b013e318204e815

Sarani, B., and Gracias, V. (2008). Hemoglobin-based Blood Substitutes and Risk of Myocardial Infarction and Death. JAMA 300, 1297-1299. doi:10.1001/ jama.300.11.1297-b

Sauaia, A., Moore, E. E., and Banerjee, A. (2008). Hemoglobin-based Blood Substitutes and Risk of Myocardial Infarction and Death. JAMA 300, 1297-1299. doi:10.1001/jama.300.11.1297-a

Scatena, R., and Giardina, B. (2001). O-raffinose-polymerised Haemoglobin. A Biochemical and Pharmacological Profile of an Oxygen Carrier. Expert Opin. Biol. Ther. 1, 121-127. doi:10.1517/14712598.1.1.121

Shonaka, T., Matsuno, N., Obara, H., Yoshikawa, R., Nishikawa, Y., Gouchi, M., et al. (2018). Application of Perfusate with Human-Derived Oxygen Carrier Solution under Subnormothermic Machine Perfusion for Donation after Cardiac Death Liver Grafts in Pigs. Transpl. Proc 50, 2821-2825. doi:10.1016/j.transproceed.2018.02.184

Shonaka, T., Matsuno, N., Obara, H., Yoshikawa, R., Nishikawa, Y., Ishihara, Y., et al. (2019). Impact of Human-Derived Hemoglobin Based Oxygen Vesicles as a Machine Perfusion Solution for Liver Donation after Cardiac Death in a Pig Model. PloS one 14, e0226183. doi:10.1371/journal.pone.0226183

Simoni, J., Simoni, G., Hartsell, A., and Feola, M. (1997). An Improved Blood Substitute. In Vivo Evaluation of its Renal Effects. ASAIO J. 43, M714-M725. American Society for Artificial Internal Organs : 1992. doi:10.1097/00002480199703000-00046

Standl, T. (2005). A New Oxygen Transport Agent. Haematologica 90, 437-438. doi: $10.3324 / \% 25 \mathrm{x}$

Standl, T., Freitag, M., Burmeister, M. A., Horn, E. P., Wilhelm, S., and Am Esch, J. S. (2003). Hemoglobin-based Oxygen Carrier HBOC-201 Provides Higher 
and Faster Increase in Oxygen Tension in Skeletal Muscle of Anemic Dogs Than Do Stored Red Blood Cells. J. Vasc. Surg. 37, 859-865. doi:10.1067/ mva.2003.127

Taverne, Y. J., de Wijs-Meijler, D., Te Lintel Hekkert, M., Moon-Massat, P. F., Dubé, G. P., Duncker, D. J., et al. (2017). Normalization of Hemoglobin-Based Oxygen Carrier-201 Induced Vasoconstriction: Targeting Nitric Oxide and Endothelin. J. Appl. Physiol. 122, 1227-1237. doi:10.1152/japplphysiol.00677.2016

Teh, E. S., Zal, F., Polard, V., Menasché, P., and Chambers, D. J. (2017). HEMO2life as a Protective Additive to Celsior Solution for Static Storage of Donor Hearts Prior to Transplantation. Artif. Cell Nanomed Biotechnol 45, 717-722. doi:10.1080/21691401.2016.1265974

Thuillier, R., Dutheil, D., Trieu, M. T., Mallet, V., Allain, G., Rousselot, M., et al. (2011). Supplementation with a New Therapeutic Oxygen Carrier Reduces Chronic Fibrosis and Organ Dysfunction in Kidney Static Preservation. Am. J. Transpl. 11, 1845-1860. doi:10.1111/j.1600-6143.2011.03614.x

Tian, T., Lindell, S. L., Henderson, S. C., and Mangino, M. J. (2009). Protective Effects of Ezrin on Cold Storage Preservation Injury in the Pig Kidney Proximal Tubular Epithelial Cell Line (LLC-PK1). Transplantation 87, 1488-1496. doi:10.1097/TP.0b013e3181a43f18

Topp, S. A., Krieg, A., Koch, A., Tidden, C. M., Ramp, U., Hohlfeld, T., et al. (2008). Hemoglobin-glutamer 200 Reduces Reperfusion Injury of the Cold Preserved Rat Liver by Induction of Heme Oxygenase-1. J. Surg. Res. Dec 150, 243-254. doi:10.1016/j.jss.2008.02.033

Toulmond, A., Slitine, F. E. I., De Frescheville, J., and Jouin, C. (1990). Extracellular Hemoglobins of Hydrothermal Vent Annelids: Structural and Functional Characteristics in Three Alvinellid Species. Biol. Bull. 179, 366-373. doi: $10.2307 / 1542329$

Tran, N. Q., Malcontenti-Wilson, C., Hammoud, S., Millar, I., Christophi, C., and Muralidharan, V. (2012). Hyperbaric Oxygen Therapy Reduces the Severity of Ischaemia, Preservation and Reperfusion Injury in a Rat Model of Liver Transplantation. HPB (Oxford) 14, 103-114. doi:10.1111/j.14772574.2011.00410.x

Tsujimura, T., Kuroda, Y., Kin, T., Avila, J. G., Rajotte, R. V., Korbutt, G. S., et al. (2002). Human Islet Transplantation from Pancreases with Prolonged Cold Ischemia Using Additional Preservation by the Two-Layer (UW Solution/ perfluorochemical) Cold-Storage Method. Transplantation 74, 1687-1691. doi:10.1097/00007890-200212270-00007

Unnikrishnan, A., Pelletier, J. P. R., Bari, S., Zumberg, M., Shahmohamadi, A., Spiess, B. D., et al. (2019). Anti-N and Anti-Do immunoglobulinGalloantibody -mediateddelayed Hemolytic Transfusion Reaction with Hyperhemolysis in Sickle Cell Disease Treated with Eculizumab and HBOC -201:case Report and Review of the Literature. Transfus.59, 1907-1910. doi:10.1111/trf.15198

van Leeuwen, O. B., de Vries, Y., Fujiyoshi, M., Nijsten, M. W. N., Ubbink, R., Pelgrim, G. J., et al. (2019). Transplantation of High-Risk Donor Livers after $E x$ Situ Resuscitation and Assessment Using Combined Hypo- and Normothermic Machine Perfusion: A Prospective Clinical Trial. Ann. Surg. Nov 270, 906-914. doi:10.1097/SLA.0000000000003540

Warnecke, G., Strüber, M., Hohlfeld, J. M., Niedermeyer, J., Sommer, S. P., and Haverich, A. (2002). Pulmonary Preservation with Bretscheider's HTK and
Celsior Solution in Minipigs. Eur. J. cardio-thoracic Surg. : official J. Eur. Assoc. Cardio-thoracic Surg. 21, 1073-1079. doi:10.1016/s1010-7940(02)00106-9

Wei, L., Wu, R. B., Yang, C. M., Zheng, S. Y., and Yu, X. Y. (2011). Cardioprotective Effect of a Hemoglobin-Based Oxygen Carrier on Cold Ischemia/reperfusion Injury. Cardiology 120, 73-83. doi:10.1159/000333106

White, C. W., Hasanally, D., Mundt, P., Li, Y., Xiang, B., Klein, J., et al. (2015). A Whole Blood-Based Perfusate Provides superior Preservation of Myocardial Function during Ex Vivo Heart Perfusion. J. Heart Lung Transplant. : official Publ. Int. Soc. Heart Transplant. 34, 113-121. doi:10.1016/j.healun.2014.09.021

York, G. B., DiGeronimo, R. J., Wilson, B. J., Cofer, B. R., Breuer, C. K., Josephs, J. D., et al. (2002). Extracorporeal Membrane Oxygenation in Piglets Using a Polymerized Bovine Hemoglobin-Based Oxygen-Carrying Solution (HBOC201). J. Pediatr. Surg. 37, 1387-1392. doi:10.1053/jpsu.2002.35374

Yoshida, H., Okuno, H., Kamoto, T., Habuchi, T., Toda, Y., Hasegawa, S., et al. (2002). Comparison of the Effectiveness of ET-Kyoto with Euro-Collins and University of Wisconsin Solutions in Cold Renal Storage. Transplantation 74, 1231-1236. doi:10.1097/00007890-200211150-00006

You, Z., Li, T., Yang, C., Wu, H., Peng, X., Zeng, Y., et al. (2011). Comparison of the Cardioprotective Effect of Deoxygenated Hemoglobin-based Oxygen Carrier (HBOC) Pretreatment and Ischemia Preconditioning. Artif. Cells Blood Substit. Immobil. Biotechnol. 39 (1), 7-11. doi:10.3109/10731199.2010.495037

Zal, F., Green, B. N., Lallier, F. H., Vinogradov, S. N., and Toulmond, A. (1997). Quaternary Structure of the Extracellular Haemoglobin of the Lugworm Arenicola marina: a Multi-Angle-Laser-Light-Scattering and ElectrosprayIonisation-Mass-Spectrometry Analysis. Eur. J. Biochem. 243, 85-92. doi:10.1111/j.1432-1033.1997.85_1a.x

Zhu, Y., Zhang, J., Wu, Y., Tian, K., Peng, X., Xiang, X., et al. (2019). Beneficial Effects of Hemoglobin-Based Oxygen Carriers on Early Resuscitation in Rats with Uncontrolled Hemorrhagic Shock. Zhonghua Wei Zhong Bing Ji Jiu Yi Xue 31, 81-86. doi:10.3760/cma.j.issn.2095-4352.2019.01.016

Conflict of Interest: The authors declare that the research was conducted in the absence of any commercial or financial relationships that could be construed as a potential conflict of interest.

Publisher's Note: All claims expressed in this article are solely those of the authors and do not necessarily represent those of their affiliated organizations, or those of the publisher, the editors, and the reviewers. Any product that may be evaluated in this article, or claim that may be made by its manufacturer, is not guaranteed or endorsed by the publisher.

Copyright (c) 2021 Cao, Wang, He, Yue, Zhao, Pan, Huang, Guo, Yin, Ma, Zhang and Huang. This is an open-access article distributed under the terms of the Creative Commons Attribution License (CC BY). The use, distribution or reproduction in other forums is permitted, provided the original author(s) and the copyright owner(s) are credited and that the original publication in this journal is cited, in accordance with accepted academic practice. No use, distribution or reproduction is permitted which does not comply with these terms. 


\section{GLOSSARY}

SOT solid organ transplantation

DCD donation after cardiac death

IRI ischemia-reperfusion injury

ECD expanded criteria donor

SCS static cold storage

ATP adenosine triphosphate

RBCs red blood cells

RBC red blood cell

PFCs perfluorochemicals

SNMP subnormothermic machine perfusion

NMP normothermic machine perfusion

HBOCs hemoglobin-based oxygen carriers

Hb hemoglobin

Mb myoglobin

MI myocardial infarction

FDA Food and Drug Administration

EA expanded access

ECMO extracorporeal membrane oxygenation

HbV hemoglobin-vesicles

PolyPHb polymerized human placenta hemoglobin

HbG hemoglobin glutamer

PLP pyridoxal phosphate

GDA glutaraldehyde

PEG polyethylene glycol

NO nitric oxide

ET endothelin

CO carbon monoxide

EC Euro-Collins

UW University of Wisconsin

HTK histidine-tryptophan-ketoglutarate

DHOPE dual hypothermic oxygenated machine perfusion

COR controlled oxygenated rewarming
MP machine perfusion

TLR2 toll-like receptor 2

TLR4 toll-like receptor 4

NF-kB nuclear factor kappa B

LVDP left ventricular development pressure

EVHP ex vivo heart perfusion

ROS reactive oxygen species

CVR coronary vascular resistance

GMP good manufacturing practice

SOD superoxide dismutase

MDA malondialdehyde

PGD primary graft dysfunction

BALF bronchoalveolar lavage fluid

$\mathrm{PaO}_{2} / \mathrm{FiO}_{2}$ arterial partial pressure of oxygen to fraction of inspired oxygen ratio

HMGB1 high mobility group box 1

RAGE advanced glycation end products

EVLP ex vivo lung perfusion

HO-1 heme oxygenase 1

SnPP Tin protoporphyrin IX dichloride

CS cold storage

Mrp2 multidrug resistance-associated protein 2

Bsep bile salt export pump

JNK c-Jun N-terminal kinase

HOPE hypothermic oxygenated machine perfusion

CSP current standard-of-care

HMP hypothermic machine perfusion

IFTA interstitial fibrosis and tubular atrophy

NGLA neutrophil gelatinase-associated lipocalin

KIM-1 kidney injury molecule-1

PRBC packed red blood cell

PolyHb polyhemoglobin

HCA hypertonic adenine citrate solution 